\title{
MAUREY'S FACTORIZATION THEORY FOR OPERATOR SPACES
}

\author{
MARIUS JUNGE AND JAVIER PARCET
}

\section{Contents}

Introduction

\section{INTRODUCTION}

In Banach space theory probabilistic techniques play a central role. For example in the local theory of Banach spaces, geometric properties of finite dimensional subspaces are proved from probabilistic inequalities. The probabilistic approach not only enriched Banach space theory, but also introduced Banach space techniques in other areas such as probability or convex geometry. A famous instance of such interplay is Maurey/Pisier's theory of type and cotype. Their results are certainly inspired by Rosenthal's work on subspaces of $L_{p}$. On the other hand, the latter is strongly influenced by Grothendieck's notion of absolutely summing maps, extended by Pietsch to $p>1$ and further developed by Lindenstrauss/Pelczynski in their fundamental work on Grothendieck's inequality.

All attempts to develop a similar theory for operator spaces have had only a limited success, so far. This is probably due to the fact that there are many, if not too many, different operator space structures on any Hilbert space. Indeed, in the local theory of Banach spaces classification results typically measure the distance of finite dimensional subspaces to Hilbert spaces and then study critical indices, such as the best type $p$ or cotype $q$ index [26, 30. Therefore, the best one can hope for is that for a given operator space there is a Hilbertian structure which allows a similar local theory in the context of operator spaces. A good illustration of this approach is Pisier's version of Dvoretzky's theorem for operator spaces [37. We will take a different approach here.

This paper is inspired by the work on the 'Grothendieck's program' for operator spaces [3, 8, 12, 41, 45. To be more precise, let us start by describing Rosenthal's theorem for subspaces of $L_{p}$ and Maurey's factorization theorem. We first recall some classical notions for a linear map $T: X \rightarrow Y$ between Banach spaces.

Junge is partially supported by the NSF DMS-0556120.

Parcet is partially supported by 'Programa Ramón y Cajal 2005'

and by Grants MTM2007-60952 and CCG07-UAM/ESP-1664, Spain. 
- $T$ has cotype $q$ if

$$
\left(\sum_{k=1}^{n}\left\|T x_{k}\right\|_{Y}^{q}\right)^{\frac{1}{q}} \leq c_{q}(T)\left(\mathbb{E}\left\|\sum_{k=1}^{n} \varepsilon_{k} x_{k}\right\|_{X}^{q}\right)^{\frac{1}{q}},
$$

- $T$ is absolutely $(q, 1)$-summing if

$$
\left(\sum_{k=1}^{n}\left\|T x_{k}\right\|_{Y}^{q}\right)^{\frac{1}{q}} \leq \pi_{q, 1}(T) \sup _{\varepsilon_{k}= \pm 1}\left\|\sum_{k=1}^{n} \varepsilon_{k} x_{k}\right\|_{X},
$$

- $T$ is $q$-summing if

$$
\left(\sum_{k=1}^{n}\left\|T x_{k}\right\|_{Y}^{q}\right)^{\frac{1}{q}} \leq \pi_{q}(T) \sup _{\|\phi\|_{X^{*} \leq 1}}\left(\sum_{k=1}^{n}\left|\left\langle\phi, x_{k}\right\rangle\right|^{q}\right)^{\frac{1}{q}} .
$$

The constants $c_{q}(T), \pi_{q, 1}(T), \pi_{q}(T)$ are the best ones for which the inequalities hold.

Rosenthal's theorem [43]. Let $X \subset L_{1}$ be infinite dimensional and let $j: X \rightarrow L_{1}$ denote the inclusion map with adjoint $j^{*}: L_{\infty} \rightarrow X^{*}$. Then, the following are equivalent:

i) $X$ embeds in $L_{p}$ for some $p>1$,

ii) $X^{*}$ has cotype $q$ for some finite $q$,

iii) $j^{*}$ is $(q, 1)$-summing for some finite $q$.

Using an adapted notion of $(q, 1)$-concave maps, Rosenthal's theorem remains true for infinite-dimensional subspaces of $L_{p}$ and $1<p<2$. The shortest way to prove Rosenthal's result is a combination of the Grothendieck/Pietsch and Maurey's factorization results. Indeed, Maurey's theorem (stated below) yields the hard inclusion iii) $\Rightarrow$ i) in Rosenthal's result. The other inclusions follows from well established facts in the theory.

Maurey's factorization theorem 29. Let $1 \leq p<q<\infty$ and let $C(K)$ denote the space of continuous functions in a compact Hausdorff space. Assume that the linear map $T: C(K) \rightarrow X$ is absolutely $(p, 1)$-summing. Then, $T$ is q-summing and the following inequality holds

$$
\pi_{q}(T) \leq c(p, q) \pi_{p, 1}(T) .
$$

This means that for any absolutely $(p, 1)$-summing map $T: C(K) \rightarrow X$, we may find a probability measure $\mu$ and a linear map $w: L_{q}(K, \mu) \rightarrow X$ such that, if $j: C(K) \rightarrow L_{q}(K, \mu)$ denotes the natural inclusion map, $T$ factorizes as

$$
T(x)=w \circ j(x) .
$$

The main result of this paper is an operator space analog of Maurey's theorem stated above and its natural generalization for mappings $T: L_{s} \rightarrow X$. We refer to 4, 40, for basic definitions on operator spaces. Motivated by Pisier's notion of a completely $q$-summing operator [39, we define a map

$$
T: X \rightarrow Y
$$

between operator spaces to be completely $(q, 1)$-summing if

$$
\pi_{q, 1}^{c b}(T)=\left\|i d \otimes T: \ell_{1} \otimes_{\min } X \rightarrow \ell_{q}(Y)\right\|_{c b}<\infty .
$$


An expert in operator space theory might think that it is more natural to take Schatten classes $S_{1}$ and $S_{q}$ instead, see Remark 3.6 below for a little discussion on this topic. Nevertheless, this weaker notion is enough to obtain the operator space analog of Maurey's factorization result.

Theorem A. If $1 \leq p<q<s<\infty$ and $X$ is an operator space, we have:

i) Let $A$ be a $C^{*}$-algebra and assume that the map $T: A \rightarrow X$ is completely $(p, 1)$-summing. Then, there exist positive elements $\delta_{1}, \delta_{2} \in L_{2 q}\left(A^{* *}\right)$ and a map $w: L_{q}\left(A^{* *}\right) \rightarrow X$ such that $T(x)=w\left(\delta_{1} x \delta_{2}\right)$ and

$$
\left\|\delta_{1}\right\|_{2 q}\|w\|_{c b}\left\|\delta_{2}\right\|_{2 q} \leq c(p, q) \pi_{p, 1}^{c b}(T) .
$$

ii) Let $\mathcal{M}$ be a von Neumann algebra and assume that the map $T: \mathcal{M} \rightarrow X^{*}$ is normal and completely $(p, 1)$-summing. Then, there exist positive elements $d_{1}, d_{2} \in L_{2 q}(\mathcal{M})$ and a map $v: L_{q}(\mathcal{M}) \rightarrow X^{*}$ such that $T(x)=v\left(d_{1} x d_{2}\right)$ and

$$
\left\|d_{1}\right\|_{2 q}\|v\|_{c b}\left\|d_{2}\right\|_{2 q} \leq c(p, q) \pi_{p, 1}^{c b}(T) .
$$

iii) Let $\mathcal{M}$ be a von Neumann algebra and assume that the map $T: L_{s}(\mathcal{M}) \rightarrow X$ is completely $(p, 1)$-summing. Then, if $1 / q=1 / s+1 / w$, there exist positive elements $d_{1}, d_{2} \in L_{2 w}(\mathcal{M})$ and a completely bounded map $v: L_{q}(\mathcal{M}) \rightarrow X$ such that $T(x)=v\left(d_{1} x d_{2}\right)$ and

$$
\left\|d_{1}\right\|_{2 w}\|v\|_{c b}\left\|d_{2}\right\|_{2 w} \leq c(p, q, s) \pi_{p, 1}^{c b}(T)
$$

Note here that the analogue of a measure on $K$ is given by a state $\phi$ on $A$. The natural analogue of the inclusion map $i d: C(K) \rightarrow L_{p}(K, \mu)$ is the positive map $j_{p}(x)=d^{1 / 2 p} x d^{1 / 2 p}$ where $d$ is the positive density of the state $\phi(x)=\operatorname{tr}(d x)$ in $L_{1}\left(A^{* *}\right)$. Despite the analogy of the results, a Banach space reader will have a hard time recognizing similarities in the proof. The main difference relies on the probabilistic part of the argument. Indeed, the new aspect of the key embedding is based on our previous work [17, 18. Let us state it here since it might be of independent interest. Let $X$ be an operator space and $\mathcal{M}$ be a von Neumann algebra. Let us say that a linear map $T: X \rightarrow L_{p}(\mathcal{M})$ is $\left(p_{1}, p_{2}\right)$-convex if

$$
k_{\left(p_{1}, p_{2}\right)}(T)=\left\|i d \otimes T: \ell_{p_{1}}(X) \rightarrow L_{p}\left(\mathcal{M} ; \ell_{p_{2}}\right)\right\|_{c b}<\infty .
$$

Theorem B. Assume that

$$
T: X \rightarrow L_{p}(\mathcal{M})
$$

is $\left(p_{1}, p_{2}\right)$-convex and $1 \leq p<q<\left(p_{1} \wedge p_{2}\right) \leq \infty$. Then, we have

$$
\left\|T \otimes i d: S_{q}(X) \rightarrow L_{p}\left(\mathcal{M} ; S_{q}\right)\right\|_{c b} \leq c\left(p, q, p_{1}, p_{2}\right) k_{\left(p_{1}, p_{2}\right)}(T) .
$$

We must emphasize that Theorems A and B hold for general von Neumann algebras. The lack of a general theory of vector-valued noncommutative $L_{p}$ spaces for arbitrary algebras forces us to start with a careful analysis of the spaces we will handle along the paper. Let us also note that in the special case $p=1$, Theorem $\mathrm{B}$ is a dual version of Theorem $\mathrm{A}$, and the corresponding notion of concavity is even slightly weaker than the assumption in Theorem A i). Our first application is of course an operator space analog of Rosenthal's theorem. Our notion of cotype here will be the following. Let $2 \leq q<\infty$ and

$$
\operatorname{Rad}_{q}(X)=\left\{\sum_{k} \varepsilon_{k} x_{k} \mid x_{k} \in X\right\} \subset L_{q}(\Sigma ; X),
$$


where the $\varepsilon_{k}$ 's are independent \pm 1 Bernoulli's on a probability space $(\Sigma, \nu)$. Let $\iota$ be determined by $\iota\left(\varepsilon_{k}\right)=\delta_{k}$, where the $\delta_{k}$ 's form the canonical basis of $\ell_{q}$. Then we say that a linear map $T: X \rightarrow Y$ between operator spaces has cb-cotype $q$ if

$$
c_{q}^{c b}(T)=\left\|\iota \otimes T: \operatorname{Rad}_{q}(X) \rightarrow \ell_{q}(Y)\right\|_{c b}<\infty .
$$

An operator space $X$ has cb-cotype $q$ if $i d_{X}$ does. We refer to [5, 27, 31, 32, for previous attempts of defining a satisfactory notion of type and cotype for operator spaces. In the following results, $p^{\prime}$ will denote the conjugate index of $p, \frac{1}{p}+\frac{1}{p^{\prime}}=1$. Rosenthal's result takes the following form in the operator space setting.

Corollary A1. If $1 \leq p<2$ and $X \subset L_{p}(\mathcal{M})$, t.f.a.e.

i) There exists $p<q<2$ such that $X^{*}$ is of cb-cotype $q^{\prime}$.

ii) There exists $p<q<2$ such that $X^{*}$ is completely $\left(q^{\prime}, 1\right)$-summing.

iii) There exists $p<q<2$ such that $X$ completely embeds into $L_{q}(\mathcal{M})$.

In the category of Banach spaces, Rosenthal's theorem was recently extended in [16 for subspaces of noncommutative $L_{p}$ spaces. Although the relation with that result is obvious, we note that Corollary A1 is not comparable since both hypotheses and conclusions are stronger. Let us continue with the example of Pisier's operator space $O H=[R, C]_{1 / 2}$. It is not too difficult to prove that the identity map on $O H$ is completely $(2,1)$ summing, see Lemma 3.1. However, we know from 12 that the strong version of the little Grothendieck inequality fails

$$
\mathcal{C B}(\mathcal{B}(\mathcal{H}), O H) \not \subset \Pi_{2}^{o}(\mathcal{B}(\mathcal{H}), O H) \text {. }
$$

Corollary A2. If $A$ is a $C^{*}$-algebra, $u: A \rightarrow O H$ is completely bounded if and only if there exist positive elements $a, b \in L_{1}\left(A^{* *}\right)$ and a cb-map $w: L_{p}\left(A^{* *}\right) \rightarrow O H$ for some (all) $2<p<\infty$ such that $u=w\left(a^{1 / 2 p} x b^{1 / 2 p}\right)$. In particular, the isomorphism $\Pi_{p^{\prime}}^{o}(O H, Y)=\Pi_{1}^{o}(O H, Y)$ holds for $2<p<\infty$ and any operator space $Y$.

We refer to [39] for the definition of the completely $p$-summing norm $\pi_{p}^{o}$ and the space $\Pi_{p}^{o}(X, Y)$ of completely $p$-summing maps $T: X \rightarrow Y$. This corollary vastly improves on the results in [14. We see that $p>2$ is sharp in this result, in contrast to what happens for Banach spaces. We end up the paper with some further applications for Fourier multipliers on discrete groups and other mappings between noncommutative $L_{p}$ spaces.

\section{VeCtoR-VALUed $L_{p}$ SPACES}

Vector-valued, noncommutative $L_{p}$ spaces where introduced by Pisier [39]. One of the main applications is a successful understanding of noncommutative square and maximal functions. We now discuss several settings for which vector-valued noncommutative $L_{p}$ spaces are defined and which will be needed below.

1.1. The hyperfinite case. In Pisier's setting, we assume that $\mathcal{M}$ is a hyperfinite von Neumann algebra and $X$ is an arbitrary operator space. For $1 \leq p<\infty$, the space $L_{p}(\mathcal{M})=\lim _{\lambda} L_{p}\left(\mathcal{M}_{\lambda}\right)$ is a norm limit of finite dimensional von Neumann subalgebras $\mathcal{M}_{\lambda}$. Therefore, it really suffices to understand vector-valued Schatten $p$-classes. If $R_{p}^{m}$ and $C_{p}^{m}$ stand for the row and column subspaces of $S_{p}^{m}$, then define

$$
S_{p}^{m}(X)=C_{p}^{m} \otimes_{h} X \otimes_{h} R_{p}^{m} .
$$


In operator space theory, the pairing $(a, b)=\operatorname{tr}\left(a^{t} b\right)$ is chosen between $S_{1}^{m}$ and $M_{m}$. With respect to the paring $\langle a, b\rangle=\operatorname{tr}(a b)$, we can reformulate the main properties as follows:

a) If $1 \leq p \leq \infty$, then

$$
\begin{aligned}
& \text { a1) }\|x\|_{L_{p}(\mathcal{M} ; X)}=\inf _{x=a y b}\|a\|_{2 p}\|y\|_{\mathcal{M} \otimes_{\min } X}\|b\|_{2 p}, \\
& \text { a2) }\|x\|_{L_{p}(\mathcal{M} ; X)}=\sup _{\|a\|_{2 p^{\prime}},\|b\|_{2_{p^{\prime}}} \leq 1}\|a x b\|_{L_{1}(\mathcal{M} ; X)} .
\end{aligned}
$$

b) If $1 \leq p<\infty, L_{p}(\mathcal{M} ; X)^{*}=L_{p^{\prime}}\left(\mathcal{M}^{\mathrm{op}} ; X^{*}\right)$ with respect to the bracket

$$
\left\langle\sum_{j} a_{j} \otimes x_{j}^{*}, \sum_{k} b_{k} \otimes x_{k}\right\rangle=\sum_{j, k} \operatorname{tr}\left(a_{j} b_{k}\right)\left\langle x_{j}^{*}, x_{k}\right\rangle .
$$

1.2. Amalgamated and conditional $L_{p}$ spaces. Let us now recall some new noncommutative function spaces from 18 which will be essential below. Let $\mathcal{M}$ be an arbitrary von Neumann algebra and let $\mathcal{R}$ stand for the matrix amplification $\mathcal{M} \bar{\otimes} \mathcal{B}\left(\ell_{2}\right)$. In what follows we shall work with indices represented in the following solid of $\mathbb{R}^{3}$

$$
\mathrm{K}=\{(1 / u, 1 / v, 1 / q) \mid 2 \leq u, v \leq \infty, 1 \leq q \leq \infty, 1 / u+1 / q+1 / v \leq 1\} .
$$

Given $1 \leq p \leq \infty$ such that $\frac{1}{p}=\frac{1}{u}+\frac{1}{q}+\frac{1}{v}$ for some $\left(\frac{1}{u}, \frac{1}{v}, \frac{1}{q}\right)$ in $\mathrm{K}$, we define the corresponding amalgamated $L_{p}$ space as the subspace $L_{\underline{u} q \underline{v}}(\mathcal{R} ; \mathcal{M})$ of $L_{p}(\mathcal{R})$ equipped with the norm

$$
\|x\|_{\underline{u} q \underline{v}}=\inf \left\{\|a\|_{L_{u}(\mathcal{M})}\|y\|_{L_{q}(\mathcal{R})}\|b\|_{L_{v}(\mathcal{M})} \mid x=a y b\right\} .
$$

We shall also be interested in the duals of amalgamated $L_{p}$ spaces. To that end given $\left(\frac{1}{u}, \frac{1}{v}, \frac{1}{p}\right) \in \mathrm{K}$ and $\frac{1}{s}=\frac{1}{u}+\frac{1}{p}+\frac{1}{v}$, we define the corresponding conditional $L_{p}$ space $L_{\bar{u} s \bar{v}}(\mathcal{R} ; \mathcal{M})$ as the completion of $L_{p}(\mathcal{R})$ with respect to the norm

$$
\|x\|_{\bar{u} s \bar{v}}=\sup \left\{\|\alpha x \beta\|_{L_{s}(\mathcal{R})} \mid\|\alpha\|_{L_{u}(\mathcal{M})},\|\beta\|_{L_{v}(\mathcal{M})} \leq 1\right\} .
$$

We refer to 18 for a more detailed exposition and note in passing that we have changed/improved our terminology for amalgamated and conditional $L_{p}$ 's. Now we collect the main complex interpolation and duality properties from [18. Let $\mathrm{K}_{0}$ denote the interior of $\mathrm{K}$. Then we have:

i) $L_{\underline{u} q \underline{v}}(\mathcal{R} ; \mathcal{M})$ is a Banach space.

ii) $L_{\underline{u_{\theta}} q_{\theta} \underline{v_{\theta}}}(\mathcal{R} ; \mathcal{M})$ is isometrically isomorphic to

$$
\left[L_{\underline{u_{0}} q_{0} \underline{v_{0}}}(\mathcal{R} ; \mathcal{M}), L_{\underline{u_{1}} q_{1} \underline{v_{1}}}(\mathcal{R} ; \mathcal{M})\right]_{\theta},
$$

with $\left(\frac{1}{u_{\theta}}, \frac{1}{q_{\theta}}, \frac{1}{v_{\theta}}\right)=\left(\frac{1-\theta}{u_{0}}+\frac{\theta}{u_{1}}, \frac{1-\theta}{q_{0}}+\frac{\theta}{q_{1}}, \frac{1-\theta}{v_{0}}+\frac{\theta}{v_{1}}\right)$.

iii) If $(1 / u, 1 / v, 1 / q) \in \mathrm{K}_{0}$ and $1-1 / p=1 / u+1 / q+1 / v$

$$
\left(L_{\underline{u} q \underline{v}}(\mathcal{R} ; \mathcal{M})\right)^{*}=L_{\bar{u} q^{\prime} \bar{v}}(\mathcal{R} ; \mathcal{M}) \text { and }\left(L_{\bar{u} q^{\prime} \bar{v}}(\mathcal{R} ; \mathcal{M})\right)^{*}=L_{\underline{u} q \underline{v}}(\mathcal{R} ; \mathcal{M}) \text {, }
$$

with respect to the antilinear duality bracket $\langle x, y\rangle=\operatorname{tr}\left(x^{*} y\right)$. 
1.3. Mixed norms I. The definition of amalgamated and conditional $L_{p}$ spaces was mainly inspired by Pisier's fundamental identities for the mixed norm spaces $L_{p}\left(\mathcal{M}_{1} ; L_{q}\left(\mathcal{M}_{2}\right)\right)$ with $\mathcal{M}_{1}$ hyperfinite. Given $1 \leq p, q \leq \infty$ and $\frac{1}{r}=\left|\frac{1}{p}-\frac{1}{q}\right|$, we have

$$
\|x\|_{L_{p}\left(L_{q}\right)}= \begin{cases}\inf \left\{\|\alpha\|_{L_{2 r}\left(\mathcal{M}_{1}\right)}\|y\|_{L_{q}\left(\mathcal{M}_{1} \bar{\otimes} \mathcal{M}_{2}\right)}\|\beta\|_{L_{2 r}\left(\mathcal{M}_{1}\right)} \mid x=\alpha y \beta\right\} & \text { if } p \leq q, \\ \sup \left\{\|\alpha x \beta\|_{L_{q}\left(\mathcal{M}_{1} \bar{\otimes} \mathcal{M}_{2}\right)} \mid\|\alpha\|_{L_{2 r}\left(\mathcal{M}_{1}\right)},\|\beta\|_{L_{2 r}\left(\mathcal{M}_{1}\right)} \leq 1\right\} & \text { if } p \geq q .\end{cases}
$$

The extension to arbitrary von Neumann algebras is a matter of regarding these spaces as amalgamated and conditional $L_{p}$ spaces. Indeed, given any von Neumann algebra $\mathcal{M}$ and $\mathcal{R}=\mathcal{M} \bar{\otimes} \mathcal{B}\left(\ell_{2}\right)$, we may define

$$
L_{p}\left(\mathcal{M} ; S_{q}\right)= \begin{cases}L_{\underline{2 r} q \underline{2 r}}(\mathcal{R} ; \mathcal{M}) & \text { if } p \leq q, \\ L_{\overline{2 r} q \overline{2 r}}(\mathcal{R} ; \mathcal{M}) & \text { if } p \geq q,\end{cases}
$$

Remark 1.1. We define an operator space structure on $L_{p}\left(\mathcal{M} ; S_{q}\right)$ by complex interpolation. It thus suffices to provide the o.s.s. of the endpoints $L_{p}\left(\mathcal{M} ; S_{q}\right)$ for $p, q \in\{1, \infty\}$. If $p=q$ the definition is obvious, while $L_{1}\left(\mathcal{M} ; S_{\infty}\right)$ embeds into the dual of $L_{\infty}\left(\mathcal{M} ; S_{1}\right)$. Hence, it just remains to understand the o.s.s. of the latter one. According to [40, we may define the operator space $L_{\infty}\left(\mathcal{M} ; S_{1}\right)$ as the quotient

$$
\mathcal{M} \otimes_{h} S_{1} \otimes_{h} \mathcal{M} / \operatorname{ker} q
$$

by the quotient map $q(a \otimes x \otimes b)=a b \otimes x$. Moreover, we also find

- Complex interpolation also gives $S_{p}^{n}\left(L_{p}\left(\mathcal{M} ; S_{q}\right)\right)=L_{p}\left(M_{n} \otimes \mathcal{M} ; S_{q}\right)$.

- The same argument provides an o.s.s. for $A\left(\ell_{1}\right)$ with $A$ any $C^{*}$-algebra.

Then we easily find that

a) If $1 \leq p \leq \infty$, then

$$
\begin{aligned}
& \text { a1) }\|x\|_{L_{p}\left(\mathcal{M} ; S_{q}^{m}\right)}=\inf _{x=a y b}\|a\|_{2 p}\|y\|_{L_{\infty}\left(\mathcal{M} ; S_{q}^{m}\right)}\|b\|_{2 p}, \\
& \text { a2) }\|x\|_{L_{p}\left(\mathcal{M} ; S_{q}^{m}\right)}=\sup _{\|a\|_{2 p^{\prime}},\|b\|_{2 p^{\prime}} \leq 1}\|a x b\|_{L_{1}\left(\mathcal{M} ; S_{q}^{m}\right)} .
\end{aligned}
$$

b) If $1 \leq p<\infty$, we have $L_{p}\left(\mathcal{M} ; S_{q}^{m}\right)^{*}=L_{p^{\prime}}\left(\mathcal{M} ; S_{q^{\prime}}^{m}\right)$.

Note that $\mathcal{M} \otimes_{\min } X$ in the hyperfinite case is replaced here by $L_{\infty}\left(\mathcal{M} ; S_{q}^{m}\right)$. It should be noticed that we still have $L_{p}\left(\mathcal{M} ; S_{q}^{m}\right)=\left[L_{p}\left(\mathcal{M} ; S_{\infty}^{m}\right), L_{p}\left(\mathcal{M}, S_{1}^{m}\right)\right]_{1 / q}$ for general von Neumann algebras, see [21, 23. It is well known since [11 that the norms of the boundary points are given by

$$
\begin{aligned}
\|x\|_{L_{p}\left(\mathcal{M} ; S_{\infty}^{m}\right)} & =\inf _{x=a y b}\|a\|_{L_{2 p}(\mathcal{M})}\|y\|_{M_{m}(\mathcal{M})}\|b\|_{L_{2 p}(\mathcal{M})}, \\
\|x\|_{L_{p}\left(\mathcal{M} ; S_{1}^{m}\right)} & =\inf _{x_{i j}=\sum_{k} a_{i k} b_{j k}}\left\|\left(\sum_{i, k} a_{i k} a_{i k}^{*}\right)^{\frac{1}{2}}\right\|_{2 p}\left\|\left(\sum_{j, k} b_{j k}^{*} b_{j k}\right)^{\frac{1}{2}}\right\|_{2 p} .
\end{aligned}
$$

Remark 1.2. We have just considered the amplification algebra $\mathcal{R}$ since we shall be mainly interested in mixed-norms with values in matrix algebras. Nevertheless at some points in this paper we will handle spaces of the form $L_{p}\left(\mathcal{M}_{1} ; L_{q}\left(\mathcal{M}_{2}\right)\right)$ for non-hyperfinite $\mathcal{M}_{j}$. In this case the notions of amalgamated and conditional $L_{p}$ spaces remains unchanged, see [18] for further details. 
1.4. Asymmetric Schatten classes. Given any von Neumann algebra $\mathcal{M}$, we write $L_{2}^{r}(\mathcal{M})$ and $L_{2}^{c}(\mathcal{M})$ to denote the row/column quantizations on $L_{2}(\mathcal{M})$ and consider the operator spaces

$$
L_{u}^{r}(\mathcal{M})=\left[\mathcal{M}, L_{2}^{r}(\mathcal{M})\right]_{\frac{2}{u}} \quad \text { and } \quad L_{v}^{c}(\mathcal{M})=\left[\mathcal{M}, L_{2}^{c}(\mathcal{M})\right]_{\frac{2}{v}} .
$$

In fact, a rigorous definition should take Kosaki's embeddings into account as done in [18, Identity (1.3)], but we shall ignore such formalities here. We have the complete isometry $L_{p}(\mathcal{M})=L_{2 p}^{r}(\mathcal{M}) \otimes_{\mathcal{M}, h} L_{2 p}^{c}(\mathcal{M})$, where $\otimes_{\mathcal{M}, h}$ stands for the $\mathcal{M}$-amalgamated Haagerup tensor product. This motivates the definition of the asymmetric spaces

$$
L_{(2 u, 2 v)}(\mathcal{M})=L_{2 u}^{r}(\mathcal{M}) \otimes_{\mathcal{M}, h} L_{2 v}^{c}(\mathcal{M})=L_{\underline{2 u} \infty \underline{2 v}}(\mathcal{M} ; \mathcal{M}) .
$$

These spaces were originally defined in [15] for finite matrix algebras, where the definition simplifies in terms of ordinary Haagerup tensors. In this case, given an arbitrary operator space $X$, we may as well consider the vector-valued space as

$$
S_{(2 u, 2 v)}^{m}(X)=C_{u}^{m} \otimes_{h} X \otimes_{h} R_{v}^{m} .
$$

Its module behavior is explained better by

$$
S_{(2 u, 2 v)}^{m}(X)=S_{(2 u, \infty)}^{m} \otimes_{M_{m}, h} S_{\infty}^{m}(X) \otimes_{M_{m}, h} S_{(\infty, 2 v)}^{m} .
$$

Again by interpolation, we find a natural o.s.s. for $L_{(2 u, 2 v)}(\mathcal{M})$ and we see that

$$
C_{u}^{n} \otimes_{h} L_{(2 u, 2 v)}(\mathcal{M}) \otimes_{h} R_{v}^{n}=L_{(2 u, 2 v)}\left(M_{n} \otimes \mathcal{M}\right) .
$$

According to [15, we have

a) If $1 \leq u, v \leq \infty$, then

$$
\begin{aligned}
& \text { a1) }\|x\|_{S_{(2 u, 2 v)}^{m}(X)}=\inf _{x=a y b}\|a\|_{2 u}\|y\|_{S_{\infty}^{m}(X)}\|b\|_{2 v}, \\
& \text { a2) }\|x\|_{S_{(2 u, 2 v)}^{m}(X)}=\sup _{\|a\|_{2 u^{\prime}},\|b\|_{2 v^{\prime}} \leq 1}\|a x b\|_{S_{1}^{m}(X)} .
\end{aligned}
$$

b) If $1 \leq u, v \leq \infty, S_{(2 u, 2 v)}^{m}(X)^{*}=S_{\left(2 u^{\prime}, 2 v^{\prime}\right)}^{m}\left(X^{*}\right)$ with respect to the bracket

$$
\left\langle\sum_{j} a_{j} \otimes x_{j}^{*}, \sum_{k} b_{k} \otimes x_{k}\right\rangle=\sum_{j, k} \operatorname{tr}\left(a_{j} b_{k}\right)\left\langle x_{j}^{*}, x_{k}\right\rangle .
$$

1.5. Mixed norms II. The next family of spaces are noncommutative $L_{p}$ spaces with values in asymmetric Schatten classes. Namely, let us recall the spaces $L_{\infty}\left(\mathcal{M} ; C_{q}\right)=\left[L_{\infty}\left(\mathcal{M} ; C_{\infty}\right), L_{\infty}\left(\mathcal{M} ; R_{\infty}\right)\right]_{1 / q}$ defined from the row/column spaces $L_{\infty}\left(\mathcal{M} ; R_{\infty}\right)=\mathcal{M} \bar{\otimes} R$ and $L_{\infty}\left(\mathcal{M} ; C_{\infty}\right)=C \bar{\otimes} \mathcal{M}$. The spaces $L_{\infty}\left(\mathcal{M} ; C_{q}\right)$ were already considered by Pisier for semifinite von Neumann algebras [36] and by Haagerup for general von Neumann algebras [6]. Define

$$
\begin{aligned}
& L_{2 p}^{r}\left(\mathcal{M} ; C_{q}\right)=L_{2 p}^{r}(\mathcal{M}) \otimes_{\mathcal{M}, h} L_{\infty}\left(\mathcal{M} ; C_{q}\right), \\
& L_{2 p}^{c}\left(\mathcal{M} ; R_{q}\right)=L_{\infty}\left(\mathcal{M} ; R_{q}\right) \otimes_{\mathcal{M}, h} L_{2 p}^{c}(\mathcal{M}) .
\end{aligned}
$$

These spaces satisfy:

i) $L_{2 p}^{r}\left(\mathcal{M} ; C_{p}\right)=C_{2 p}\left(L_{2 p}(\mathcal{M})\right)$ isometrically.

ii) $L_{2 p_{\theta}}^{r}\left(\mathcal{M} ; C_{q_{\theta}}\right)=\left[L_{2 p_{0}}^{r}\left(\mathcal{M} ; C_{q_{0}}\right), L_{2 p_{1}}^{r}\left(\mathcal{M} ; C_{q_{1}}\right)\right]_{\theta}$ isometrically. 
and analogous properties hold for the adjoint spaces. Indeed, the second property follows from a nowadays standard interpolation technique originated in [36] and further developed in [18, 44. The first property is clear for $p=\infty$ and it then suffices by interpolation to consider the case $p=1$. Again, this is standard by applying Pisier's factorization trick in [39]. If $\frac{1}{s}=\left|\frac{1}{p}-\frac{1}{q}\right|$, the norm can written as follows

$$
\|x\|_{L_{2 p}^{r}\left(\mathcal{M} ; C_{q}\right)}= \begin{cases}\inf _{x=\alpha y}\|\alpha\|_{2 s}\|y\|_{C_{2 q}\left(L_{2 q}(\mathcal{M})\right)} & \text { if } p \leq q, \\ \sup _{\|\alpha\|_{2 s} \leq 1}\|\alpha x\|_{C_{2 q}\left(L_{2 q}(\mathcal{M})\right)} & \text { if } p \geq q .\end{cases}
$$

If $1 \leq p \leq q_{1} \wedge q_{2} \leq \infty$ and $1 \leq s_{1}, s_{2} \leq \infty$ satisfy $\frac{1}{s_{j}}=\frac{1}{p}-\frac{1}{q_{j}}$ and $\mathcal{R}=\mathcal{M} \bar{\otimes} \mathcal{B}\left(\ell_{2}\right)$, we set

$$
L_{p}\left(\mathcal{M} ; C_{q_{1}} \otimes_{h} R_{q_{2}}\right)=L_{2 p}^{r}\left(\mathcal{M} ; C_{q_{1}}\right) \otimes_{\mathcal{M}, h} L_{2 p}^{c}\left(\mathcal{M} ; R_{q_{2}}\right) .
$$

By interpolation, it is compatible with the symmetric case given in Paragraph 1.3 .

Remark 1.3. Connes' characterization of hyperfiniteness can be rephrased by the condition $L_{\infty}(\mathcal{M} ; O H) \simeq \mathcal{M} \otimes_{\min } O H$, see 38. Thus in general we have to accept that the norms considered so far are different, but consistent. Namely, we have seen that the asymmetric/nonhyperfinite norms generalize the symmetric/hyperfinite ones respectively. Thus, there should be no ambiguity of what definition is being used along the text.

Remark 1.4. If $A$ is a $C^{*}$-algebra, consider the norm

$$
\left\|\left(x_{k}\right)\right\|_{A\left(\ell_{1}\right)}=\inf _{x_{k}=\sum_{j} a_{k j} b_{k j}}\left\|\left(\sum_{j, k} a_{j k} a_{k j}^{*}\right)^{\frac{1}{2}}\right\|\left\|\left(\sum_{j, k} b_{j k}^{*} b_{k j}\right)^{\frac{1}{2}}\right\| .
$$

Replacing $A$ by $M_{m}(A)$, we see that $A\left(\ell_{1}\right) \subset \ell_{1} \otimes_{\min } A$ is a complete contraction and according to an unpublished work of Haagerup, this is an isometry only for $C^{*}$-algebras with Lance's weak expectation property. At any rate, we see that every completely $(p, 1)$-summing map $T: A \rightarrow X$ satisfies

$$
\left\|i d \otimes T: A\left(\ell_{1}\right) \rightarrow \ell_{p}(X)\right\|_{c b} \leq \pi_{(p, 1)}^{c b}(T) .
$$

Indeed, for Theorem A i) only this weaker assumption of $(p, 1)$-concavity is required. This concavity is the cb-version of Pisier's notion of $(p, 1) C^{*}$-summability in 35 .

Remark 1.5. In the hyperfinite and asymmetric cases we considered arbitrary operator spaces and specific von Neumann algebras. In the mixed-norm cases the situation is the opposite. There exists an intermediate notion of $L_{p}(\mathcal{M} ; X)$ valid for QWEP von Neumann algebras and operator spaces contained in any $C^{*}$-algebra with the local lifting property. The notion was developed in 13 and is based on the hyperfinite theory replacing norm approximations by ultraproducts. Some arguments in this paper could be slightly simplified if we restricted to work over QWEP von Neumann algebras.

\section{Key PROBABiListic estimates}

In this section we use the theory of vector-valued $L_{p}$ spaces in connection with convexifying operators. This leads to a change of density which will be crucial for our proof of Maurey's theorem. 
2.1. A cb-embedding for $S_{q}(X)$. Let us consider a weight function $w$ indexed over the integers $\mathbb{Z}$ and define the Hilbert space $\ell_{2}(w)$ determined by the following norm

$$
\left\|\sum_{n} w_{n} \delta_{n}\right\|_{\ell_{2}(w)}=\left(\sum_{n} w_{n}\left|\alpha_{n}\right|^{2}\right)^{\frac{1}{2}}
$$

If $\ell_{2}^{r}(w)$ and $\ell_{2}^{c}(w)$ denote the row/column o.s.s. on $\ell_{2}(w)$, we set

$$
\ell_{2}^{r_{p}}(w)=\left[\ell_{2}^{r}(w), \ell_{2}^{c}(w)\right]_{\frac{1}{p}} \quad \text { and } \quad \ell_{2}^{c_{p}}(w)=\left[\ell_{2}^{c}(w), \ell_{2}^{r}(w)\right]_{\frac{1}{p}} .
$$

Most of the time, our weights will be of the form $w_{n}=\lambda^{n}$ for some $\lambda>1$. In that cases we will write $w_{\lambda}$ and $\ell_{2}\left(w_{\lambda}\right)$ instead. Our first step will be a description of $S_{q}(X)$ closely related to Xu's characterization [47 of $R_{q}$ and $C_{q}$. Although it also follows from a more general argument in [22], we give here a concrete approach for completeness. In what follows we shall write $\alpha \lesssim \beta$ to indicate the existence of an absolute constant $c$ such that $\alpha \leq c \beta$. We begin with a well-known observation comparing the $J$ and $K$ methods as in [12, 47.

Lemma 2.1. Let $A$ and $B$ be non-singular positive operators on a Hilbert space $\mathcal{H}$ and assume further than $A$ and $B$ commute. If $0<\theta<1$ and $\lambda>1$, let us consider the constants

$$
c_{1}(\lambda, \theta)=\sqrt{\frac{1}{\lambda^{\theta}-1}+\frac{1}{\lambda^{1-\theta}-1}} \quad, \quad c_{2}(\lambda, \theta)=\sqrt{\frac{1}{1-\lambda^{-\theta}}+\frac{1}{1-\lambda^{-(1-\theta)}}} .
$$

Then, the equivalence $c_{1}(\lambda, \theta) \alpha \lesssim \beta \lesssim c_{2}(\lambda, \theta) \alpha$ holds with

$$
\begin{aligned}
\alpha & =\left\|A^{\theta} B^{1-\theta} x\right\|_{\mathcal{H}}, \\
\beta & =\inf _{x=y_{n}+z_{n}}\left(\sum_{n \in \mathbb{Z}} \lambda^{n(1-\theta)}\left\|A y_{n}\right\|_{\mathcal{H}}^{2}\right)^{\frac{1}{2}}+\left(\sum_{n \in \mathbb{Z}} \lambda^{-n \theta}\left\|B z_{n}\right\|_{\mathcal{H}}^{2}\right)^{\frac{1}{2}} .
\end{aligned}
$$

$A$ duality argument also gives $c_{1}(\lambda, \theta) \beta^{\prime} \lesssim \alpha^{\prime} \lesssim c_{2}(\lambda, \theta) \beta^{\prime}$ with

$$
\begin{aligned}
\alpha^{\prime} & =\left\|A^{1-\theta} B^{\theta} x\right\|_{\mathcal{H}}, \\
\beta^{\prime} & =\inf _{A^{1-2 \theta} B^{2 \theta-1} x=\sum_{n} z_{n}}\left(\sum_{n \in \mathbb{Z}} \lambda^{-n(1-\theta)}\left\|A z_{n}\right\|_{\mathcal{H}} 2\right)^{\frac{1}{2}}+\left(\sum_{n \in \mathbb{Z}} \lambda^{n \theta}\left\|B z_{n}\right\|_{\mathcal{H}}^{2}\right)^{\frac{1}{2}} .
\end{aligned}
$$

Proof. By simultaneous diagonalization, it suffices to prove the first assertion for diagonal operators $A=D_{a}$ and $B=D_{b}$. Then it is clear that the term $\beta$ is equivalent to

$$
\inf _{x_{k}=y_{n k}+z_{n k}}\left(\sum_{n \in \mathbb{Z}} \lambda^{n(1-\theta)} \sum_{k \in \mathbb{N}}\left|a_{k}\right|^{2}\left|y_{n k}\right|^{2}+\sum_{n \in \mathbb{Z}} \lambda^{-n \theta} \sum_{k \in \mathbb{N}}\left|b_{k}\right|^{2}\left|z_{n k}\right|^{2}\right)^{\frac{1}{2}} .
$$

This equals

$$
\left(\sum_{k \in \mathbb{N}} \inf _{x_{k}=y_{n k}+z_{n k}}\left[\sum_{n \in \mathbb{Z}} \lambda^{n(1-\theta)}\left|a_{k} y_{n k}\right|^{2}+\sum_{n \in \mathbb{Z}} \lambda^{-n \theta}\left|b_{k} z_{n k}\right|^{2}\right]\right)^{\frac{1}{2}} .
$$

Thus, it suffices to prove the assertion for $k$ fixed and then we may even assume that $x_{k}=1$ by normalization. This reduces the assertion to scalars and we therefore claim that

$$
c_{1}(\lambda, \theta) a^{\theta} b^{1-\theta} \lesssim \mathcal{B} \lesssim c_{2}(\lambda, \theta) a^{\theta} b^{1-\theta}
$$


for $a, b>0$ and

$$
\mathcal{B}=\inf _{1=\gamma_{n}+\rho_{n}}\left(\sum_{n \in \mathbb{Z}} \lambda^{n(1-\theta)}\left|a \gamma_{n}\right|^{2}+\sum_{n \in \mathbb{Z}} \lambda^{-n \theta}\left|b \rho_{n}\right|^{2}\right)^{\frac{1}{2}} .
$$

Let us start with an easy observation

$$
\inf _{1=\gamma+\rho} \delta|\gamma|^{2}+\sigma|\rho|^{2}=\inf _{0 \leq t \leq 1} \delta t^{2}+\sigma(1-t)^{2}=\frac{\delta \sigma}{\delta+\sigma} \sim \min (\delta, \sigma)
$$

holds for all $\delta, \sigma>0$. Going back to our claim, since $\left|\gamma_{n}\right|+\left|\rho_{n}\right| \geq 1$, it therefore suffices to consider $\gamma_{n}$ and $\rho_{n}$ positive in the right hand side above. This leads to the following estimate

$$
\begin{aligned}
\mathcal{B}^{2} & =\inf _{1=\gamma_{n}+\rho_{n}} \sum_{n \in \mathbb{Z}}\left[a^{2} \lambda^{n(1-\theta)}\left|\gamma_{n}\right|^{2}+b^{2} \lambda^{-n \theta}\left|\rho_{n}\right|^{2}\right] \sim \sum_{n \in \mathbb{Z}} \min \left(a^{2} \lambda^{n(1-\theta)}, b^{2} \lambda^{-n \theta}\right) \\
& =\sum_{\lambda^{-n} \geq a^{2} / b^{2}} a^{2} \lambda^{n(1-\theta)}+\sum_{\lambda^{-n}<a^{2} / b^{2}} b^{2} \lambda^{-n \theta}=a^{2} \frac{\lambda^{n_{0}(1-\theta)}}{1-\lambda^{-(1-\theta)}}+b^{2} \frac{\lambda^{-\left(n_{0}+1\right) \theta}}{1-\lambda^{-\theta}} .
\end{aligned}
$$

Here $n_{0}$ is chosen so that $\lambda^{-\left(n_{0}+1\right)}<a^{2} / b^{2} \leq \lambda^{-n_{0}}$ and this gives

$$
c_{1}(\lambda, \theta)^{2} a^{2 \theta} b^{2(1-\theta)} \lesssim \mathcal{B}^{2} \lesssim c_{2}(\lambda, \theta)^{2} a^{2 \theta} b^{2(1-\theta)} .
$$

Hence, the first assertion follows. To prove the second assertion, given a positive non-singular operator $L$ acting on $\mathcal{H}$, we denote by $\mathcal{H}_{L}$ the Hilbert space equipped with the norm

$$
\|x\|_{\mathcal{H}_{L}}=\|L x\|_{\mathcal{H}} .
$$

By the first assertion, we know that $\mathcal{H}_{A^{\theta} B^{1-\theta}}$ is isomorphic (up to the constants $\left.c_{j}(\lambda, \theta)\right)$ to the subspace of constant sequences in $\ell_{2}\left(\lambda^{1-\theta} ; \mathcal{H}_{A}\right)+\ell_{2}\left(\lambda^{-\theta} ; \mathcal{H}_{B}\right)$. Since $\mathcal{H}_{A^{\theta} B^{1-\theta}}$ is a Hilbert space, it is isometric to its dual. In particular, recalling that

$$
A^{1-\theta} B^{\theta} x=A^{\theta} B^{1-\theta}\left(A^{1-2 \theta} B^{2 \theta-1} x\right),
$$

we find that its norm in $\mathcal{H}$ is equivalent to the norm of $A^{1-2 \theta} B^{2 \theta-1} x$ in the quotient of $\ell_{2}\left(\lambda^{-(1-\theta)} ; \mathcal{H}_{A}\right) \cap \ell_{2}\left(\lambda^{\theta} ; \mathcal{H}_{B}\right)$ by the subspace of mean zero sequences. Writing this down we obtain the second assertion. The proof is complete.

To continue, we need to introduce Xu's terminology in 47. We will only define the column spaces, but we shall freely use below the row analogs which are defined in the obvious way. Let

$\ell_{2}\left(w ; \ell_{2}\right)=\ell_{2}\left(\mathbb{Z}, w ; \ell_{2}(\mathbb{N})\right) \quad$ with norm $\left\|\sum_{n, k} x_{n k} \otimes \delta_{n k}\right\|=\left(\sum_{n \in \mathbb{Z}} w_{n} \sum_{k \in \mathbb{N}}\left|x_{n k}\right|^{2}\right)^{\frac{1}{2}}$.

Define $\ell_{2}\left(w_{\lambda} ; \ell_{2}\right)$ similarly, let $\ell_{2}^{c_{p}}\left(w_{\lambda} ; \ell_{2}\right)=\left[\ell_{2}^{c}\left(w_{\lambda} ; \ell_{2}\right), \ell_{2}^{r}\left(w_{\lambda} ; \ell_{2}\right)\right]_{\frac{1}{p}}$ and set

$$
\mathcal{G}_{c_{p}, c_{q}}^{K}\left(w_{\lambda}, \theta\right)=\ell_{2}^{c_{p}}\left(w_{\lambda}^{-\theta} ; \ell_{2}\right)+\ell_{2}^{c_{q}}\left(w_{\lambda}^{1-\theta} ; \ell_{2}\right) \quad \text { with } \quad w_{\lambda}^{\eta}=w_{\lambda^{\eta}}
$$

Let $\mathcal{C}_{c_{p}, c_{q}}^{K}\left(w_{\lambda}, \theta\right)$ denote the subspace of $\mathbb{Z}$-constant sequences. Using the bracket

$$
\left\langle\left(a_{n k}\right),\left(b_{n k}\right)\right\rangle=\sum_{n \in \mathbb{Z}} \sum_{k \in \mathbb{N}} \bar{a}_{n k} b_{n k},
$$

the dual spaces are

$$
\left(\mathcal{G}_{c_{p}, c_{q}}^{K}\left(w_{\lambda}, \theta\right)\right)^{*}=\mathcal{G}_{c_{p^{\prime}}, c_{q^{\prime}}}^{J}\left(w_{\lambda}^{-1}, \theta\right)
$$




$$
\left(\mathcal{C}_{c_{p}, c_{q}}^{K}\left(w_{\lambda}, \theta\right)\right)^{*}=\mathcal{C}_{c_{p^{\prime}}, c_{q^{\prime}}}^{J}\left(w_{\lambda}^{-1}, \theta\right)
$$

with operator space structures given by

$$
\begin{aligned}
\left\|\sum_{n \in \mathbb{Z}} \sum_{k=1}^{\infty} x_{n k} \otimes e_{(n, k), 1}\right\|_{M_{m}\left(\mathcal{G}_{J}\right)} & =\max \left\{n_{p}(x), n_{q}(x)\right\} \\
\left\|\sum_{n \in \mathbb{Z}} \sum_{k=1}^{\infty} x_{n k} \otimes e_{(n, k), 1}+\mathcal{C}_{K}^{\perp}\right\|_{M_{m}\left(\mathcal{C}_{J}\right)} & =\inf _{\sum_{n} x_{n k}-z_{n k}=0} \max \left\{n_{p}(z), n_{q}(z)\right\}
\end{aligned}
$$

where the norms $n_{p}(\xi)$ and $n_{q}(\xi)$ are given by

$$
\begin{aligned}
& n_{p}(\xi)=\left\|\sum_{n \in \mathbb{Z}} \lambda^{n \theta / 2} \sum_{k=1}^{\infty} \xi_{n k} \otimes e_{(n, k), 1}\right\|_{M_{m}\left(C_{p^{\prime}}\right)}, \\
& n_{q}(\xi)=\left\|\sum_{n \in \mathbb{Z}} \lambda^{-n(1-\theta) / 2} \sum_{k=1}^{\infty} \xi_{n k} \otimes e_{(n, k), 1}\right\|_{M_{m}\left(C_{q^{\prime}}\right)} .
\end{aligned}
$$

The following result is closely related to [46, Section 2]. However we have to review the argument in order to understand the generalization presented below.

Lemma 2.2. If $p_{0}<q<p_{1}$ with $\frac{1}{q}=\frac{1-\theta}{p_{0}}+\frac{\theta}{p_{1}}$ and $\lambda>1$, then

$$
R_{q} \simeq_{c b} \mathcal{C}_{r_{p_{0}}, r_{p_{1}}}^{K}\left(w_{\lambda}, \theta\right) \quad \text { and } \quad C_{q} \simeq_{c b} \mathcal{C}_{c_{p_{0}}, c_{p_{1}}}^{K}\left(w_{\lambda}, \theta\right) .
$$

The constant of these complete isomorphisms only depend on $\lambda$ and $\theta$.

Proof. Since both cb-isomorphisms are proved in the same way, we only argue with column spaces. Let us first show that the inclusion $\mathcal{C}_{c_{p_{0}}, c_{p_{1}}}^{K}\left(w_{\lambda}, \theta\right) \subset C_{q}$ is completely bounded. We recall the o.s.s. of $C_{q}$ from the main result in 44

$$
\left\|\sum_{k=1}^{\infty} x_{k} \otimes e_{k, 1}\right\|_{M_{m}\left(C_{q}\right)}=\sup _{\|a\|_{S_{2 q}^{m}}^{m},\|b\|_{S_{2 q^{\prime}}^{m}} \leq 1}\left(\sum_{k=1}^{\infty}\left\|a x_{k} b\right\|_{2}^{2}\right)^{\frac{1}{2}} .
$$

We may clearly assume that $a$ and $b$ are positive and invertible. Let us denote by $\mathcal{L}_{a}(x)=a x$ and $\mathcal{R}_{b}(x)=x b$ the left/right actions. We define $A=\mathcal{L}_{a^{q / p_{1}}} \mathcal{R}_{b^{q^{\prime}} / p_{1}^{\prime}}$ and $B=\mathcal{L}_{a^{q / p_{0}}} \mathcal{R}_{b^{q^{\prime} / p_{0}^{\prime}}}$. Then we apply Lemma 2.1 to $x=\sum_{k} x_{k} \otimes e_{k, 1}$ and deduce that we have

$$
\left(\sum_{k=1}^{\infty}\left\|a x_{k} b\right\|_{2}^{2}\right)^{\frac{1}{2}}=\left\|A^{\theta} B^{1-\theta} x\right\|_{2} \lesssim c_{1}(\lambda, \theta)^{-1} \inf _{x_{k}=y_{n k}+z_{n k}}\left\{n_{y}, n_{z}\right\}
$$

where

$$
\begin{aligned}
& n_{y}=\left(\sum_{n \in \mathbb{Z}} \lambda^{-n \theta} \sum_{k=1}^{\infty}\left\|a^{q / p_{0}} y_{n k} b^{q^{\prime} / p_{0}^{\prime}}\right\|_{2}^{2}\right)^{\frac{1}{2}}, \\
& n_{z}=\left(\sum_{n \in \mathbb{Z}} \lambda^{n(1-\theta)} \sum_{k=1}^{\infty}\left\|a^{q / p_{1}} z_{n k} b^{q^{\prime} / p_{1}^{\prime}}\right\|_{2}^{2}\right)^{\frac{1}{2}} .
\end{aligned}
$$

Using again the o.s.s. of $\ell_{2}^{c_{p_{0}}}\left(w_{\lambda}^{-\theta} ; \ell_{2}\right)$ and $\ell_{2}^{c_{p_{1}}}\left(w_{\lambda}^{1-\theta} ; \ell_{2}\right)$ as above, we get

$$
\left(\sum_{k=1}^{\infty}\left\|a x_{k} b\right\|_{2}^{2}\right)^{\frac{1}{2}} \lesssim c_{1}(\lambda, \theta)^{-1}\left\|\mathbf{1}_{\mathbb{Z}} \otimes x\right\|_{M_{m}\left(\mathcal{C}_{K}\right)}
$$


where $\mathbf{1}_{\mathbb{Z}}=\sum_{n \in \mathbb{Z}} \delta_{n}$ is the constant- 1 sequence in $\mathbb{Z}$. Let us now show that

$$
\mathcal{C}_{c_{p_{0}^{\prime}}, c_{p_{1}^{\prime}}}^{J}\left(w_{\lambda}^{-1}, \theta\right) \subset C_{q^{\prime}} .
$$

Indeed, arguing by homogeneity we assume that $\sum_{n, k} x_{n k} \otimes e_{(n, k), 1}$ satisfies

$$
\left\|\sum_{n, k} x_{n k} \otimes e_{(n, k), 1}\right\|_{M_{m}\left(\mathcal{C}_{J}\right)}<1 .
$$

That is, we may find $\left(z_{n k}\right)$ such that $\sum_{n} x_{n k}=\sum_{n} z_{n k}$ and

$$
\max \left\{\left\|\sum_{n, k} \lambda^{\frac{n \theta}{2}} z_{n k} \otimes e_{(n, k), 1}\right\|_{M_{m}\left(C_{p_{0}^{\prime}}\right)}\left\|\sum_{n, k} \lambda^{\frac{-n(1-\theta)}{2}} z_{n k} \otimes e_{(n, k), 1}\right\|_{M_{m}\left(C_{p_{1}^{\prime}}\right)}\right\} \leq 1 .
$$

Taking $z_{n}=\sum_{k} z_{n k} \otimes e_{k}$ and

$$
A=\mathcal{L}_{a^{q^{\prime} / p_{1}^{\prime}}} \mathcal{R}_{b^{q / p_{1}}} \quad \text { and } \quad B=\mathcal{L}_{a^{q^{\prime} / p_{0}^{\prime}}} \mathcal{R}_{b^{q / p_{0}}},
$$

we observe that

$$
\begin{aligned}
\sum_{n \in \mathbb{Z}} \lambda^{-n(1-\theta)}\left\|A z_{n}\right\|_{2}^{2}+\sum_{n \in \mathbb{Z}} \lambda^{n \theta}\left\|B z_{n}\right\|_{2}^{2} & \\
& =\sum_{n, k} \lambda^{-n(1-\theta)}\left\|a^{q^{\prime} / p_{1}^{\prime}} z_{n k} b^{q / p_{1}}\right\|_{2}^{2}+\sum_{n, k} \lambda^{n \theta}\left\|a^{q^{\prime} / p_{0}^{\prime}} z_{n k} b^{q / p_{0}}\right\|_{2}^{2} \leq 2
\end{aligned}
$$

holds by our assumption. According to Lemma 2.1 $\xi=A^{2 \theta-1} B^{1-2 \theta} \sum_{n} z_{n}$ satisfies $\left\|A^{1-\theta} B^{\theta} \xi\right\|_{2} \lesssim c_{2}(\lambda, \theta)$ and for $x=\sum_{k} x_{k} \otimes e_{k, 1}$ with $x_{k}=\sum_{n} x_{n k}=\sum_{n} z_{n k}$, we have

$$
\left(\sum_{k=1}^{\infty}\left\|a x_{k} b\right\|_{2}^{2}\right)^{\frac{1}{2}}=\left\|A^{\theta} B^{1-\theta} x\right\|_{2}=\left\|A^{1-\theta} B^{\theta} A^{2 \theta-1} B^{1-2 \theta}\left(\sum_{n \in \mathbb{Z}} z_{n}\right)\right\|_{2} \lesssim c_{2}(\lambda, \theta) .
$$

Therefore, duality yields $C_{q} \subset \mathcal{C}_{c_{p_{0}}, c_{p_{1}}}^{K}\left(w_{\lambda}, \theta\right)$ and the assertion follows.

Our next step is to construct a complete embedding of $S_{q}(X)$ into a 4-term sum. Together with Proposition 2.6 below, this cb-embedding will be the key towards the main result in this section. Let $\mathcal{K}_{p, q}(w ; X)$ be defined by

$$
S_{p}(X)+C_{p} \otimes_{h} X \otimes_{h} \ell_{2}^{r_{q}}(w)+\ell_{2}^{c_{q}}(w) \otimes_{h} X \otimes_{h} R_{p}+\ell_{2}^{c_{q}}(w) \otimes_{h} X \otimes_{h} \ell_{2}^{r_{q}}(w) .
$$

Let us write $\mathcal{K}_{p, q}(w)$ for the same space when $X=\mathbb{C}$ and $\mathcal{K}_{p, q}\left(w_{\lambda} ; X\right) / \mathcal{K}_{p, q}\left(w_{\lambda}\right)$ for exponential sequences. Here it is important to recall that we will be considering weights $w$ on the index set $\mathbb{Z} \times \mathbb{N}$ which are constant on the $\mathbb{N}$-component, so that (using the terminology above) another description for this space could be

$$
\mathcal{K}_{p, q}(w ; X)=\left[C_{p}(\mathbb{Z} \times \mathbb{N})+\ell_{2}^{c_{q}}\left(w ; \ell_{2}\right)\right] \otimes_{h} X \otimes_{h}\left[R_{p}(\mathbb{Z} \times \mathbb{N})+\ell_{2}^{r_{q}}\left(w ; \ell_{2}\right)\right] .
$$

In the following result, we study a map $S_{q}(X) \rightarrow \mathcal{K}_{p_{0}, p_{1}}(w ; X)$ of the form

$$
\sum_{k, \ell=1}^{\infty} e_{k, 1} \otimes x_{k \ell} \otimes e_{1, \ell} \mapsto \sum_{i, j=-\infty}^{\infty} w_{i j}\left(p_{0}, p_{1}, q\right) \sum_{k, \ell=1}^{\infty} e_{i, 1} \otimes e_{k, 1} \otimes x_{k \ell} \otimes e_{1, \ell} \otimes e_{1, j} .
$$

Just to shorten the notation, we change the order of tensors and write

$$
x \mapsto\left(\sum_{i, j=-\infty}^{\infty} w_{i j}\left(p_{0}, p_{1}, q\right) e_{i j}\right) \otimes x .
$$

With this terminology, we have $\mathbf{1}_{\mathbb{Z}} \otimes \mathbf{1}_{\mathbb{Z}}=\sum_{i, j \in \mathbb{Z}} e_{i j}$ for $\mathbf{1}_{\mathbb{Z}}=\sum_{n \in \mathbb{Z}} \delta_{n}$ as above. 
Proposition 2.3. If $p_{0}<q<p_{1}$ with $\frac{1}{q}=\frac{1-\theta}{p_{0}}+\frac{\theta}{p_{1}}$ and $\lambda>1$, then

$$
u: x \in S_{q}(X) \mapsto\left(\sum_{i, j=-\infty}^{\infty} \lambda^{-(i+j) \theta / 2} e_{i j}\right) \otimes x \in \mathcal{K}_{p_{0}, p_{1}}\left(w_{\lambda} ; X\right)
$$

is a completely isomorphic embedding with constants depending only on $(\lambda, \theta)$.

Proof. According to Lemma 2.2, the mappings

$$
\begin{aligned}
& x \in C_{q} \quad \mapsto \mathbf{1}_{\mathbb{Z}} \otimes x \in \mathcal{C}_{c_{p_{0}}, c_{p_{1}}}^{K}\left(w_{\lambda}, \theta\right), \\
& x \in R_{q} \mapsto \mathbf{1}_{\mathbb{Z}} \otimes x \in \mathcal{C}_{r_{p_{0}}, r_{p_{1}}}^{K}\left(w_{\lambda}, \theta\right),
\end{aligned}
$$

are cb-isomorphisms. Recalling that $\mathbf{1}_{\mathbb{Z}} \otimes \mathbf{1}_{\mathbb{Z}}=\sum_{i, j \in \mathbb{Z}} e_{i j}$, we get

$$
x \in C_{q} \otimes_{h} X \otimes_{h} R_{q} \mapsto\left(\sum_{i, j} e_{i j}\right) \otimes x \in \mathcal{C}_{c_{p_{0}}, c_{p_{1}}}^{K}\left(w_{\lambda}, \theta\right) \otimes_{h} X \otimes_{h} \mathcal{C}_{r_{p_{0}}, r_{p_{1}}}^{K}\left(w_{\lambda}, \theta\right)
$$

a complete isomorphism. The right hand side inherits its o.s.s. from

$$
\begin{gathered}
\mathcal{G}_{c_{p_{0}}, c_{p_{1}}}^{K}\left(w_{\lambda}, \theta\right) \otimes_{h} X \otimes_{h} \mathcal{G}_{r_{p_{0}}, r_{p_{1}}}^{K}\left(w_{\lambda}, \theta\right)=\sum_{i, j=1,2} \mathcal{U}_{i} \otimes_{h} X \otimes_{h} \mathcal{V}_{j} \\
\mathcal{U}_{1}=\ell_{2}^{c_{p_{0}}}\left(w_{\lambda}^{-\theta} ; \ell_{2}\right), \mathcal{U}_{2}=\ell_{2}^{c_{p_{1}}}\left(w_{\lambda}^{1-\theta} ; \ell_{2}\right), \mathcal{V}_{1}=\ell_{2}^{r_{p_{0}}}\left(w_{\lambda}^{-\theta} ; \ell_{2}\right), \mathcal{V}_{2}=\ell_{2}^{r_{p_{1}}}\left(w_{\lambda}^{1-\theta} ; \ell_{2}\right) .
\end{gathered}
$$

Thus, is suffices to show that the map

$$
z \in \sum_{i, j=1,2} \mathcal{U}_{i} \otimes_{h} X \otimes_{h} \mathcal{V}_{j} \mapsto\left(\sum_{i \in \mathbb{Z}} \lambda^{-i \theta / 2} e_{i i}\right) z\left(\sum_{j \in \mathbb{Z}} \lambda^{-j \theta / 2} e_{j j}\right) \in \mathcal{K}_{p_{0}, p_{1}}\left(w_{\lambda} ; X\right)
$$

is a complete embedding, in this case with constants independent on $\lambda$ and $\theta$. Moreover, since both spaces are the sum of 4 spaces indexed respectively by $\left(p_{0}, p_{0}\right)$, $\left(p_{0}, p_{1}\right),\left(p_{1}, p_{0}\right)$ and $\left(p_{1}, p_{1}\right)$, it clearly suffices to check our claim term by term. However, this later fact follows from repeated use of the complete isometries

$$
\begin{aligned}
& z \in \ell_{2}^{c_{p}}\left(w_{\lambda_{1}} ; \ell_{2}\right) \otimes_{h} X \otimes_{h} \ell_{2}^{r_{q}}\left(w_{\lambda_{2}} ; \ell_{2}\right) \\
& \mapsto\left(\sum_{i \in \mathbb{Z}} \lambda_{1}^{i / 2} e_{i i}\right) z\left(\sum_{j \in \mathbb{Z}} \lambda_{2}^{j / 2} e_{j j}\right) \in C_{p} \otimes_{h} X \otimes_{h} R_{q},
\end{aligned}
$$

with $\lambda_{1}, \lambda_{2} \in\left\{\lambda^{-\theta}, \lambda^{1-\theta}\right\}$ and $p, q \in\left\{p_{0}, p_{1}\right\}$. Details are left to the reader.

Remark 2.4. The cb-embedding of $L_{p}(\mathcal{M})$ into a von Neumann algebra predual from [17, 18] can be described by means of the map $u: L_{p}(\mathcal{M}) \rightarrow \mathcal{K}_{1,2}\left(w_{\lambda}\right)$ defined on $\mathcal{M} \bar{\otimes} \mathcal{B}\left(\ell_{2}(\mathbb{Z})\right)$ with the weight given by $\lambda$. Indeed, it suffices to apply the Poisson map from [18, Section 8.2] (a suitable average of sums of independent copies which embeds $L_{1}+L_{2}^{r+c}(w)$ in $L_{1}$, for a suitable strictly seminfinite weight $w$ ) with coefficients in $O H$ in order to embed $\mathcal{K}_{1,2}\left(w_{\lambda}\right)$ into some $L_{1}(\mathcal{A})$.

We shall also need an extended form of the embedding of Proposition 2.3 for arbitrary von Neumann algebras. More concretely, that we have an isomorphic embedding $L_{p_{0}}\left(\mathcal{M} ; S_{q}\right) \rightarrow L_{p_{0}}\left(\mathcal{M} ; \mathcal{K}_{p_{0}, p_{1}}\left(w_{\lambda}\right)\right)$. Fortunately, we only need this in the scalar case $X=\mathbb{C}$, something that simplifies our approach very much. Our first task is to define the space $L_{p_{0}}\left(\mathcal{M} ; \mathcal{K}_{p_{0}, p_{1}}(w)\right)$ appropriately. We have

$$
\mathcal{K}_{p_{0}, p_{1}}(w)=\left[C_{p_{0}}+C_{p_{1}}(w)\right] \otimes_{h}\left[R_{p_{0}}+R_{p_{1}}(w)\right]
$$


where the row/column spaces are taken in the index set $\mathbb{Z} \times \mathbb{N}$ and the weight $w$ is constant on the $\mathbb{N}$-component. Recall that in Section 1 we have defined the spaces $L_{2 p}^{r}\left(\mathcal{M} ; C_{q}\right)$ and $L_{2 p}^{c}\left(\mathcal{M} ; R_{q}\right)$ and the same definition is valid for the weighted row/column spaces. Moreover, we may define

$$
\begin{aligned}
& L_{2 p}^{r}\left(\mathcal{M} ; C_{q_{1}}\left(w_{1}\right)+C_{q_{2}}\left(w_{2}\right)\right)=L_{2 p}^{r}\left(\mathcal{M} ; C_{q_{1}}\left(w_{1}\right)\right)+L_{2 p}^{r}\left(\mathcal{M} ; C_{q_{2}}\left(w_{2}\right)\right), \\
& L_{2 p}^{c}\left(\mathcal{M} ; R_{q_{1}}\left(w_{1}\right)+R_{q_{2}}\left(w_{2}\right)\right)=L_{2 p}^{c}\left(\mathcal{M} ; R_{q_{1}}\left(w_{1}\right)\right)+L_{2 p}^{c}\left(\mathcal{M} ; R_{q_{1}}\left(w_{2}\right)\right),
\end{aligned}
$$

for arbitrary weights by viewing them as both embedded into the space of sequences with values in $L_{2 p}(\mathcal{M})$, and thereby defining the sum by taking the corresponding quotients. This allows us to consider

$$
L_{p_{0}}\left(\mathcal{M} ; \mathcal{K}_{p_{0}, p_{1}}(w)\right)=L_{2 p_{0}}^{r}\left(\mathcal{M} ; C_{p_{0}}+C_{p_{1}}(w)\right) \otimes_{\mathcal{M} ; h} L_{2 p_{0}}^{c}\left(\mathcal{M} ; R_{p_{0}}+R_{p_{1}}(w)\right)
$$

with norm given by

$$
\inf _{x_{i j}=\sum_{k} \alpha_{i k} \beta_{k j}}\left\|\left(\alpha_{i k}\right)\right\|_{L_{2 p_{0}}^{r}\left(\mathcal{M} ; C_{p_{0}}+C_{p_{1}}(w)\right) \otimes_{h} R}\left\|\left(\beta_{k j}\right)\right\|_{C \otimes_{h} L_{2 p_{0}}^{c}\left(\mathcal{M} ; R_{p_{0}}+R_{p_{1}}(w)\right)} .
$$

Proposition 2.5. If $p_{0}<q<p_{1}$ with $\frac{1}{q}=\frac{1-\theta}{p_{0}}+\frac{\theta}{p_{1}}$ and $\lambda>1$, then

$$
u: x \in L_{p_{0}}\left(\mathcal{M} ; S_{q}\right) \mapsto\left(\sum_{i, j=-\infty}^{\infty} \lambda^{-(i+j) \theta / 2} e_{i j}\right) \otimes x \in L_{p_{0}}\left(\mathcal{M} ; \mathcal{K}_{p_{0}, p_{1}}\left(w_{\lambda}\right)\right)
$$

is a complete isomorphic embedding with constants depending only on $(\lambda, \theta)$.

Proof. Lemma 2.2 remains valid here as well, i.e. we have

$$
\begin{array}{llll}
L_{2 p_{0}}^{r}\left(\mathcal{M} ; \mathcal{C}_{c_{p_{0}}, c_{p_{1}}}^{K}\left(w_{\lambda}, \theta\right)\right) & \simeq_{c b} & L_{2 p_{0}}^{r}\left(\mathcal{M} ; C_{q}\right), \\
L_{2 p_{0}}^{c}\left(\mathcal{M} ; \mathcal{C}_{r_{p_{0}}, r_{p_{1}}}^{K}\left(w_{\lambda}, \theta\right)\right) & \simeq_{c b} & L_{2 p_{0}}^{c}\left(\mathcal{M} ; R_{q}\right) .
\end{array}
$$

Indeed, by the factorization properties of the spaces involved it really suffices to prove this for $p_{0}=\infty$, and then the exact same argument in Lemma 2.2 applies since the key formula is the operator space structure of $C_{q}$, which according to [6. 36] is still valid for arbitrary von Neumann algebras

$$
\left\|\sum_{k} x_{k} \otimes e_{k, 1}\right\|_{L_{\infty}\left(\mathcal{M} ; C_{q}\right)}=\sup _{\|a\|_{L_{2 q}(\mathcal{M})},\|b\|_{L_{2 q^{\prime}}(\mathcal{M})} \leq 1}\left(\sum_{k}\left\|a x_{k} b\right\|_{L_{2}(\mathcal{M})}^{2}\right)^{\frac{1}{2}} .
$$

Since we have

$$
L_{p_{0}}\left(\mathcal{M} ; S_{q}\right)=L_{2 p_{0}}^{r}\left(\mathcal{M} ; C_{q}\right) \otimes_{\mathcal{M}, h} L_{2 p_{0}}^{c}\left(\mathcal{M} ; R_{q}\right),
$$

an element in $L_{p_{0}}\left(\mathcal{M} ; S_{q}\right)$ factorizes as a product of two elements in $L_{2 p_{0}}^{r}\left(\mathcal{M} ; C_{q}\right)$ and $L_{2 p_{0}}^{c}\left(\mathcal{M} ; R_{q}\right)$ respectively. Thus, we deduce it can be written as a product of two sums from

$$
L_{2 p_{0}}^{r}\left(\mathcal{M} ; \mathcal{C}_{c_{p_{0}}, c_{p_{1}}}^{K}\left(w_{\lambda}, \theta\right)\right) \text { and } \quad L_{2 p_{0}}^{c}\left(\mathcal{M} ; \mathcal{C}_{r_{p_{0}}, r_{p_{1}}}^{K}\left(w_{\lambda}, \theta\right)\right)
$$

respectively. Arguing as in Proposition 2.3. we see that $u$ is bounded. To prove the converse, we observe that a norm estimate for $u(x)$ means a factorization of the form $x_{k \ell}=\sum_{m} \lambda^{i \theta / 2} \alpha_{i k, m} \beta_{m, j \ell} \lambda^{j \theta / 2}$ valid for all $i, j \in \mathbb{Z}$ and with

$$
\begin{aligned}
\left(\alpha_{i k, m}\right) & \in L_{2 p_{0}}^{r}\left(\mathcal{M} ; C_{p_{0}}+C_{p_{1}}\left(w_{\lambda}\right)\right) \otimes_{h} R, \\
\left(\beta_{m, j \ell}\right) & \in C \otimes_{h} L_{2 p_{0}}^{c}\left(\mathcal{M} ; R_{p_{0}}+R_{p_{1}}\left(w_{\lambda}\right)\right) .
\end{aligned}
$$

We may rewrite this as

$$
x_{k \ell}=a_{i k} b_{j \ell}
$$


where $a_{i k}=\lambda^{i \theta / 2} \sum_{m} \alpha_{i k, m} \otimes e_{1 m}$ and $b_{j \ell}=\lambda^{j \theta / 2} \sum_{m} \beta_{j \ell} \otimes e_{m 1}$. We may also assume by approximation that we are only dealing with finitely many nonzero entries $x_{k \ell}$ with full left and right support in a finite von Neumann algebra $\mathcal{M}$. Let $e_{j \ell}$ be the left support of $b_{j \ell}$. Then we deduce from $a_{i k} b_{j \ell}=x_{k \ell}=a_{i^{\prime} k} b_{j \ell}$ that we have $a_{i k} e_{j l}=a_{i^{\prime} k} e_{j l}$. This holds for all indices $j, \ell$ and hence for $e=\vee e_{j \ell}$ we find $a_{i k} e=a_{i^{\prime} k} e$ for all $k$ and $i \neq i^{\prime}$. Taking $v_{k}=a_{i k} e$, we deduce

$$
x_{k \ell}=v_{k} b_{j \ell}
$$

for all $j$. Similarly, let $f$ be supremum of the right supports of the $v_{k}$ 's. As above we deduce that $f b_{j \ell}=f b_{j^{\prime} \ell}$ for all $\ell$ and $j \neq j^{\prime}$. Thus we may define $w_{\ell}=f b_{j \ell}$ and obtain a factorization

$$
x_{k \ell}=v_{k} w_{\ell}
$$

such that $v_{k}=a_{i k} e$ and $w_{\ell}=f b_{j \ell}$. Since the space $L_{2 p_{0}}^{r}\left(\mathcal{R} ; C_{p_{0}}+C_{p_{1}}\left(w_{\lambda}\right)\right)$ is a right $\mathcal{R}$-module and $L_{2 p_{0}}^{c}\left(\mathcal{R} ; R_{p_{0}}+R_{p_{1}}\left(w_{\lambda}\right)\right)$ is a left $\mathcal{R}$-module, we may now apply the announced extension of Lemma 2.2 and deduce that

$$
\left(v_{k}\right) \in L_{2 p_{0}}^{r}\left(\mathcal{M} ; C_{q}\right) \quad, \quad\left(w_{\ell}\right) \in L_{2 p_{0}}^{c}\left(\mathcal{M} ; R_{q}\right) .
$$

This implies $x=\left(x_{k l}\right) \in L_{p_{0}}\left(\mathcal{M} ; S_{q}\right)$ and hence $u$ is an isomorphism. Tensoring with another copy of $L_{p_{0}}\left(M_{n}\right)$ does not change constants in this argument and hence $u$ is indeed a complete isomorphism. The proof is complete.

2.2. Change of density. We need an alternative description of $L_{p}\left(\mathcal{M} ; \mathcal{K}_{q_{1}, q_{2}}(w)\right)$ according to another description of the space $L_{p}\left(\mathcal{M} ; C_{q_{1}} \otimes_{h} R_{q_{2}}\right)$. Namely if we take $\frac{1}{p}=\frac{1}{s_{j}}+\frac{1}{q_{j}}$ and $\frac{1}{q}=\frac{1}{2 q_{1}}+\frac{1}{2 q_{2}}$, we have the Banach space isometry

$$
L_{p}\left(\mathcal{M} ; C_{q_{1}} \otimes_{h} R_{q_{2}}\right)=L_{\underline{2 s_{1}} \underline{2 s_{2}}}(\mathcal{R} ; \mathcal{M}) .
$$

This follows again by complex interpolation. In particular, $L_{p}\left(\mathcal{M} ; \mathcal{K}_{q_{1}, q_{2}}(w)\right)$ is Banach space isomorphic to a 4-term sum of amalgamated $L_{p}$ spaces. Certain embedding in [18 for these spaces will be essential in the following change of density argument. Recall the notion of $\left(p_{1}, p_{2}\right)$-convex maps $T: X \rightarrow L_{p}(\mathcal{M})$ from the Introduction.

Proposition 2.6. Let $1 \leq p<p_{1} \wedge p_{2} \leq \infty$ and

$$
\alpha=\left(\frac{1}{p}-\frac{1}{p_{2}}\right) /\left(\frac{1}{p}-\frac{1}{p_{1}}\right) .
$$

If $T: X \rightarrow L_{p}(\mathcal{M})$ is $\left(p_{1}, p_{2}\right)$-convex and $w$ is any weight, then

$$
T \otimes i d: \mathcal{K}_{p, p_{1}}(w ; X) \rightarrow L_{p}\left(\mathcal{M} ; \mathcal{K}_{p, p_{2}}\left(w^{\alpha}\right)\right) \quad \text { with } \quad\left(w^{\alpha}\right)_{n}=\left(w_{n}\right)^{\alpha}
$$

is completely bounded and its cb-norm can be estimated by $c\left(p, p_{2}\right) k_{\left(p_{1}, p_{2}\right)}(T)$.

Proof. We may and will assume that $X$ is finite dimensional. Given natural numbers $m, n \in \mathbb{N}$, consider a faithful state $\phi_{m}$ on $M_{m}$ with density $d_{\phi_{m}}$. We may regard the density $d_{n \phi_{m}}$ of $n \phi_{m}$ as a diagonal operator whose entries form a weight on the index set $\{1,2, \ldots, m\}$. Define

$$
\mathcal{K}_{p, q}^{n}\left(\phi_{m} ; X\right)=\left[\ell_{2}^{c_{p}}\left(d_{n \phi_{m}}^{\frac{1}{p}}\right)+\ell_{2}^{c_{q}}\left(d_{n \phi_{m}}^{\frac{1}{q}}\right)\right] \otimes_{h} X \otimes_{h}\left[\ell_{2}^{r_{p}}\left(d_{n \phi_{m}}^{\frac{1}{p}}\right)+\ell_{2}^{r_{q}}\left(d_{n \phi_{m}}^{\frac{1}{q}}\right)\right] .
$$


As above, the expression $\mathcal{K}_{p, q}^{n}\left(\phi_{m}\right)$ will be reserved for the scalar-valued case. The space $\mathcal{K}_{p, q}^{n}\left(\phi_{m} ; X\right)$ can be written as a 4 -term sum of asymmetric $L_{p}$ spaces as in [15, 17, 18]. Namely, if we consider the asymmetric spaces

$$
L_{(2 p, 2 q)}\left(\phi_{m} ; X\right)=\ell_{2}^{c_{p}}\left(d_{\phi_{m}}^{\frac{1}{p}}\right) \otimes_{h} X \otimes_{h} \ell_{2}^{r_{q}}\left(d_{\phi_{m}}^{\frac{1}{q}}\right),
$$

we have

$$
\left\|\sum_{i, j=1}^{m} x_{i j} \otimes e_{i j}\right\|_{L_{(2 p, 2 q)}\left(n \phi_{m} ; X\right)}=n^{\frac{1}{2 p}+\frac{1}{2 q}}\left\|d_{\phi_{m}}^{\frac{1}{2 p}}\left(\sum_{i, j=1}^{m} x_{i j} \otimes e_{i j}\right) d_{\phi_{m}}^{\frac{1}{2 q}}\right\|_{C_{p}^{m} \otimes_{h} X \otimes_{h} R_{q}^{m}} .
$$

This gives a description of $\mathcal{K}_{p, q}^{n}\left(\phi_{m} ; X\right)$ in terms of asymmetric Schatten classes.

Consider the $n$-fold free product

$$
\mathcal{A}_{n}=\left(M_{m}, \phi_{m}\right)^{* n} .
$$

According to [12, we know that $\mathcal{A}_{n}$ is QWEP. In particular, it is very well-known the existence of a normal $*$-homomorphism $\rho$ and a normal conditional expectation $\mathcal{E}$ as follows

$$
\rho: \mathcal{A}_{n} \rightarrow\left(\prod_{\mathcal{U}} S_{1}\right)^{*} \text { and } \mathcal{E}:\left(\prod_{\mathcal{U}} S_{1}\right)^{*} \rightarrow \mathcal{A}_{n}
$$

We also know that we have $L_{p}$ extensions $\rho_{p}$ and $\mathcal{E}_{p}$ for $1 \leq p<\infty$. Let us denote by $\pi_{j}: M_{m} \rightarrow \mathcal{A}_{n}$ the $j$-th coordinate map. Then $\rho \pi_{j}: M_{m} \rightarrow\left[\prod_{\mathcal{U}} S_{1}\right]^{*}$ is a $*$-homomorphism. Following an argument of Kirchberg, we observe that by Kaplansky's density theorem the unit ball of $\prod_{\mathcal{U}} S_{\infty}$ is dense in the strong and strong* topology of $\left[\prod_{\mathcal{U}} S_{1}\right]^{*}$. Let $B=\ell_{\infty}^{s t *}\left(\mathcal{I}, \prod S_{\infty}\right)$ the $C^{*}$-algebra of all strong and strong* converging families. Then $\left[\prod_{\mathcal{U}} S_{1}\right]^{*}$ is a quotient of $B$. Since $M_{m}$ is nuclear, we can apply the Choi-Effros lifting theorem [2, Theorem 3.10] for the maps $\pi_{j}$ and find nets $v_{s, j}: M_{m} \rightarrow \prod_{\mathcal{U}} S_{\infty}$ of completely positive and contractive maps such that $\left(v_{s, j}\right)$ converges to $\rho \pi_{j}$ in the strong and strong* topologies. Let us consider the maps

$$
\begin{gathered}
u_{1}: x \in \mathcal{K}_{p, p_{1}}^{n}\left(\phi_{m} ; X\right) \mapsto \sum_{j=1}^{n} \rho_{p} \pi_{j}(x) \otimes \delta_{j} \in \prod_{\mathcal{U}} S_{p}\left(\ell_{p_{1}}^{n}(X)\right), \\
u_{2}: x \in L_{p}\left(\mathcal{M} ; \mathcal{K}_{p, p_{2}}^{n}\left(\phi_{m}\right)\right) \mapsto \sum_{j=1}^{n}\left(i d_{L_{p}(\mathcal{M})} \otimes \pi_{j}\right)(x) \otimes \delta_{j} \in L_{p}\left(\mathcal{A}_{n} \bar{\otimes} \mathcal{M} ; \ell_{p_{2}}^{n}\right) .
\end{gathered}
$$

We claim that $u_{1}$ is completely contractive and $u_{2}$ is an embedding. Let us note that $u_{1}$ is also a cb-embedding, a fact which will not be needed nor proved in this paper. The proof that $u_{2}$ is an embedding (defining $L_{p}\left(\mathcal{M} ; \mathcal{K}_{p, p_{2}}^{n}\left(\phi_{m}\right)\right.$ ) as indicated before the statement of this result) was given in Theorem 7.3 and Remark 7.4 of 18. Moreover, we know that $u_{2}$ is a complete contraction while the cb-norm of its inverse is controlled by a constant $c\left(p, p_{2}\right)$, see Remark 2.8 below for more on the value of $c\left(p, p_{2}\right)$. For the first part of the claim, let us show that

$$
\left\|u_{1}(x)\right\|_{\prod_{\mathcal{U}} S_{p}\left(\ell_{p_{1}}^{n}(X)\right)} \leq n^{\frac{1}{p}}\|x\|_{L_{(2 p, 2 p)}\left(\phi_{m} ; X\right)} .
$$

In fact, we will only prove this inequality since the remaining ones for the terms associated to $\left(2 p, 2 p_{1}\right),\left(2 p_{1}, 2 p\right)$ and $\left(2 p_{1}, 2 p_{1}\right)$ are similar. Indeed, we refer the 
reader to [15, Proposition 3.5] for the exact same argument. Since $p<p_{1}$, we have

$$
\|u(x)\|_{\prod_{\mathcal{U}} S_{p}\left(\ell_{p_{1}}^{n}(X)\right)} \leq\left(\sum_{j=1}^{n}\left\|\rho_{p} \pi_{j}(x)\right\|_{\prod_{\mathcal{U}} S_{p}(X)}^{p}\right)^{\frac{1}{p}} .
$$

Therefore, it suffices to consider a fixed component $j$. We may write $x=a y b^{*}$ such that $a, b \in L_{2 p}^{r}\left(\phi_{m}\right)$ are of norm 1 and $\|x\|_{L_{(2 p, 2 p)}\left(\phi_{m} ; X\right)} \sim\|y\|_{M_{m}(X)}$. Then, the element $y_{s, j}$ defined by

$$
y_{s, j}=\left(v_{s, j} \otimes i d_{X}\right)(y) \in \prod_{\mathcal{U}} S_{\infty}(X)
$$

satisfies $\left\|y_{s, j}\right\| \leq\|y\|_{M_{m}(X)}$. Moreover, the strong convergence guarantees the norm convergence of $\lim _{s} \rho_{2 p} \pi_{j}(a) y_{s, j} \rho_{2 p} \pi_{j}(b)=\rho_{2 p} \pi_{j}(a) \rho \pi_{j}(y) \rho_{2 p} \pi_{j}(b)=\rho_{p} \pi_{j}(x)$ (see 20] for further details) and we obtain

$$
\left\|\rho_{p} \pi_{j}(x)\right\|_{\prod_{\mathcal{U}} S_{p}(X)} \leq\|x\|_{L_{(2 p, 2 p)}\left(\phi_{m} ; X\right)} .
$$

Since the same inequality holds after tensorizing with the identity on $S_{p}$, this proves our claim. On the other hand, using the $\left(p_{1}, p_{2}\right)$-convexity of $T$ in conjunction with the contractivity of $u_{1}$, we deduce

$$
\begin{array}{r}
\left\|\sum_{j=1}^{n} \rho_{p}(\underbrace{\pi_{j} \otimes i d_{X}\left(d_{n \phi_{m}}^{1 / 2 p}\left(i d_{M_{m}} \otimes T(x)\right) d_{n \phi_{m}}^{1 / 2 p}\right)}_{\pi_{j}(T x) \text { for short }}) \otimes \delta_{j}\right\|_{\prod_{\mathcal{U}} S_{p}\left(L_{p}\left(\mathcal{M} ; \ell_{p_{2}}^{n}\right)\right)} \\
\leq k_{\left(p_{1}, p_{2}\right)}(T)\|x\|_{\mathcal{K}_{p, p_{1}}^{n}\left(\phi_{m} ; X\right) .}
\end{array}
$$

Moreover, we may understand this as a cb-inequality, which remains true after tensorizing with $i d_{S_{p}}$. Then we recall from [18, Chapter 3] that the space $L_{p}\left(\ell_{p_{2}}\right)$ is stable under the conditional expectation

$$
\mathcal{E}_{p}: \prod_{\mathcal{U}} S_{p}\left(L_{p}\left(\mathcal{M} ; \ell_{p_{2}}^{n}\right)\right) \rightarrow L_{p}\left(\mathcal{A}_{n} \bar{\otimes} \mathcal{M} ; \ell_{p_{2}}^{n}\right) .
$$

Therefore, we have proved that

$$
\left\|\mathcal{E}_{p} u_{1} T: \mathcal{K}_{p, p_{1}}^{n}\left(\phi_{m} ; X\right) \rightarrow L_{p}\left(\mathcal{A}_{n} \bar{\otimes} \mathcal{M} ; \ell_{p_{2}}^{n}\right)\right\|_{c b} \leq k_{\left(p_{1}, p_{2}\right)}(T) .
$$

Note that the range of $\mathcal{E}_{p} u_{1} T$ is still of the form

$$
\mathcal{E}_{p} u_{1} T(x)=\sum_{j=1}^{n} \pi_{j}(T x) \otimes \delta_{j} .
$$

This means in particular that $\mathcal{E}_{p} u_{1} T$ maps $\mathcal{K}_{p, p_{1}}^{n}\left(\phi_{m} ; X\right)$ in the range of

$$
u_{2}\left[L_{p}\left(\mathcal{M} ; \mathcal{K}_{p, p_{2}}^{n}\left(\phi_{m}\right)\right)\right] \text {. }
$$

Thus we obtain

$$
\left\|T \otimes i d: \mathcal{K}_{p, p_{1}}^{n}\left(\phi_{m} ; X\right) \rightarrow L_{p}\left(\mathcal{M} ; \mathcal{K}_{p, p_{2}}^{n}\left(\phi_{m}\right)\right)\right\|_{c b} \lesssim c\left(p, p_{2}\right) k_{\left(p_{1}, p_{2}\right)}(T) .
$$

Let us now prove the assertion. First we may replace $\phi_{m}$ by the state $\phi_{m} \otimes \tau_{\ell}$ on $M_{m \ell}$ where $\tau_{\ell}$ is the normalized trace on $M_{\ell}$. Then we note that the space of elements $x \otimes e$, with $e$ a fixed projection satisfying $\tau_{\ell}(e)=\gamma$, is simultaneously complemented in all the asymmetric spaces $L_{(2 p, 2 q)}$ considered. Thus, we restrict our attention to this subspace. Moreover, we clearly have

$$
n^{\frac{1}{2 p}+\frac{1}{2 q}}\|x \otimes e\|_{L_{(2 p, 2 q)}\left(\phi_{m} \otimes \tau_{\ell} ; X\right)}=\left\|d_{n \gamma \phi_{m}}^{\frac{1}{2 p}} x d_{n \gamma \phi_{m}}^{\frac{1}{2 q}}\right\|_{C_{p}^{m} \otimes_{h} X \otimes_{h} R_{q}^{m}} .
$$


By [15, Lemma 1.2], tensorizing with $i d_{S_{(2 p, 2 q)}}$ we obtain a complete isometry

$$
x \otimes e \in \mathcal{K}_{p, p_{1}}^{n}\left(\phi_{m} \otimes \tau_{\ell} ; X\right) \mapsto d_{n \gamma \phi_{m}}^{\frac{1}{2 p}} x d_{n \gamma \phi_{m}}^{\frac{1}{2 p}} \in \mathcal{K}_{p, p_{1}}\left(w_{\lambda} ; X\right)
$$

with $w_{\lambda}=\left(n \gamma d_{\phi_{m}}\right)^{\frac{1}{p_{1}}-\frac{1}{p}}$. A similar argument leads to the complete isometry

$$
T x \otimes e \in L_{p}\left(\mathcal{M} ; \mathcal{K}_{p, p_{2}}^{n}\left(\phi_{m} \otimes \tau_{\ell}\right)\right) \mapsto d_{n \gamma \phi_{m}}^{\frac{1}{2 p}} T x d_{n \gamma \phi_{m}}^{\frac{1}{2 p}} \in L_{p}\left(\mathcal{M} ; \mathcal{K}_{p, p_{2}}\left(w_{\mu}\right)\right)
$$

with $\mu=\left(n \gamma d_{\phi_{m}}\right)^{\frac{1}{p_{2}}-\frac{1}{p}}=w^{\alpha}$. This implies the assertion for $w=\left(n \gamma \phi_{m}\right)^{\frac{1}{p_{1}}-\frac{1}{p}}$. It just remains to show that the general case follows from this one. Indeed, by approximation it clearly suffices to show it for $w$ being a weight on $\{1,2, \ldots, m\}$ as far as we see that the constants are independent of $m$. Therefore, we have to see that every $w$ supported on $\{1,2, \ldots, m\}$ can be obtained in this form. Given such a weight $w$, we consider the functional on $M_{m}$ given by

$$
\psi_{m}\left(\sum_{i, j=1}^{m} \alpha_{i j} e_{i j}\right)=\sum_{k=1}^{m} w_{k}^{\frac{p p_{1}}{p-p_{1}}} \alpha_{k k}
$$

and the state $\phi_{m}$ defined by $\psi_{m}=\psi_{m}\left(\mathbf{1}_{M_{m}}\right) \phi_{m}$. Let us set $n=\left[\psi_{m}\left(\mathbf{1}_{M_{m}}\right)\right]+1$ where [.] stands for the integer part. Let $0<\gamma<1$ be determined by the relation $n \gamma=\psi_{m}\left(\mathbf{1}_{M_{m}}\right)$. We may assume by approximation that $\gamma$ is a rational number. Let $\tau_{\ell}$ be the normalized trace on $M_{\ell}$. Taking $\ell$ large enough, we may consider a projection $e$ in $M_{\ell}$ satisfying $\tau_{\ell}(e)=\gamma$. Hence, the embedding

$$
x \in\left(M_{m}, \phi_{m}\right) \mapsto x \otimes e \in\left(M_{m \ell}, \phi_{m} \otimes \tau_{\ell}\right)
$$

produces the desired identification $w=\left(n \gamma d_{\phi_{m}}\right)^{\frac{1}{p_{1}}-\frac{1}{p}}$. The proof is complete.

Remark 2.7. The embedding of the algebra $\mathcal{A}_{n}$ into an ultraproduct algebra is the key tool used in [13] to generalize vector-valued noncommutative $L_{p}$ spaces to QWEP algebras and such notion underlies the proof of Proposition 2.6. Note however that we do not need at any rate to require $\mathcal{M}$ to be QWEP.

Remark 2.8. According to Remarks 2.2 and 5.7 of [19], the value of the constant $c\left(p, p_{2}\right)$ above remains uniformly bounded in $p$ and $p_{2}$ as far as $\left(p, p_{2}\right) \nsim(1, \infty)$. In that case, we only know that it is controlled by $1+\frac{p_{2}-p}{p p_{2}+p-p_{2}}$. Note that this singularity near $(1, \infty)$ seems to be removable since the corresponding complete embedding holds at the point $(1, \infty)$.

Remark 2.9. Although not needed for our purposes in this paper, let us point a generalization of Proposition 2.6 for potential applications. Only for this remark we shall write $L_{p}[\mathcal{M} ; X]$ to denote the generalization of $L_{p}(\mathcal{M} ; X)$ for $\mathrm{QWEP}$ von Neumann algebras in [13. Assume that $1 \leq s \leq u \wedge v \leq u \vee v<p_{1} \wedge p_{2} \leq \infty$ and set $\beta=\left(\frac{1}{s}-\frac{1}{p_{2}}\right) /\left(\frac{1}{u}-\frac{1}{p_{1}}\right)$. If the map $T: X \rightarrow L_{v}(\mathcal{M})$ is $\left(p_{1}, p_{2}\right)$-convex and $w$ is any weight, then

$$
T \otimes i d: \mathcal{K}_{u, p_{1}}(w ; X) \rightarrow L_{v}\left(\mathcal{M} ; \mathcal{K}_{s, p_{2}}\left(w^{\beta}\right)\right)
$$

is completely bounded and its cb-norm can be estimated by $c\left(s, p_{2}\right) k_{\left(p_{1}, p_{2}\right)}(T)$. The proof follows the same pattern. Indeed, arguing as above we know that the mapping $\mathcal{E}_{u} T u_{1}: \mathcal{K}_{u, p_{1}}^{n}\left(\phi_{m} ; X\right) \rightarrow L_{u}\left[\mathcal{A}_{n} ; L_{v}\left(\mathcal{M} ; \ell_{p_{2}}^{n}\right)\right]$ is completely bounded. Moreover, we also have complete contractions

$$
L_{u}\left[\mathcal{A}_{n} ; L_{v}\left(\mathcal{M} ; \ell_{p_{2}}^{n}\right)\right] \rightarrow L_{s}\left[\mathcal{A}_{n} ; L_{v}\left(\mathcal{M} ; \ell_{p_{2}}^{n}\right)\right] \rightarrow L_{v}\left[\mathcal{M} ; L_{s}\left(\mathcal{A}_{n} ; \ell_{p_{2}}^{n}\right)\right]
$$


given by the identity map. The first one follows from the fact that $s \leq u$ and $\mathcal{A}_{n}$ is a noncommutative probability space. The second one follows from Minkowski's inequality since $s \leq v$. Then, we use again the embedding

$$
L_{v}\left(\mathcal{M} ; \mathcal{K}_{s, p_{2}}^{n}\left(\phi_{m}\right)\right) \rightarrow L_{v}\left[\mathcal{M} ; L_{s}\left(\mathcal{A}_{n} ; \ell_{p_{2}}^{n}\right)\right]
$$

to conclude

$$
\left\|T \otimes i d: \mathcal{K}_{u, p_{1}}^{n}\left(\phi_{m} ; X\right) \rightarrow L_{v}\left(\mathcal{M} ; \mathcal{K}_{s, p_{2}}^{n}\left(\phi_{m}\right)\right)\right\|_{c b} \lesssim c\left(s, p_{2}\right) k_{\left(p_{1}, p_{2}\right)}(T) .
$$

The change of density in this case is given by

$$
w=\left(n \gamma d_{\phi_{m}}\right)^{\frac{1}{p_{1}}-\frac{1}{u}} \quad \text { and } \quad \mu=\left(n \gamma d_{\phi_{m}}\right)^{\frac{1}{p_{2}}-\frac{1}{s}} .
$$

Thus, it turns out that $\mu=w^{\beta}$ for our choice of $\beta$. This completes the argument.

Now we are ready for the key embedding of this paper.

Proof of Theorem B. Let

$$
\frac{1}{q}=\frac{1-\theta}{p}+\frac{\theta}{p_{1}}=\frac{1-\eta}{p}+\frac{\eta}{p_{2}}
$$

and $\lambda>1$. Then we have the identity

$$
\alpha \eta=\frac{\frac{1}{p}-\frac{1}{p_{2}}}{\frac{1}{p}-\frac{1}{p_{1}}} \frac{\frac{1}{p}-\frac{1}{q}}{\frac{1}{p}-\frac{1}{p_{2}}}=\frac{\frac{1}{p}-\frac{1}{q}}{\frac{1}{p}-\frac{1}{p_{1}}}=\theta,
$$

where $\alpha$ is the real number defined in Proposition 2.6, Let

$$
u_{\theta, \lambda}: S_{q}(X) \rightarrow \mathcal{K}_{p, p_{1}}\left(w_{\lambda} ; X\right) \quad \text { and } \quad u_{\eta, \mu}: S_{q} \rightarrow \mathcal{K}_{p, p_{2}}\left(w_{\mu}\right)
$$

be the cb-embeddings given by Proposition 2.3 . Taking $\mu=\lambda^{\alpha}$, we note that

$$
(T \otimes i d) u_{\theta, \lambda}=\left(u_{\eta, \mu} \otimes i d_{L_{p}(\mathcal{M})}\right)(T \otimes i d) .
$$

Indeed, we deduce from $\mu^{\eta}=\lambda^{\alpha \eta}=\lambda^{\theta}$ that

$$
\begin{array}{r}
\left(u_{\eta, \mu} \otimes i d_{L_{p}(\mathcal{M})}\right)(T \otimes i d)(x)=\left(\sum_{i, j=-\infty}^{\infty} \mu^{-(i+j) \eta / 2} e_{i j}\right) \otimes T(x) \\
=\left(\sum_{i, j=-\infty}^{\infty} \lambda^{-(i+j) \theta / 2} e_{i j}\right) \otimes T(x)=(T \otimes i d) u_{\theta, \lambda}(x) .
\end{array}
$$

According to Proposition 2.6, we know that

$$
T \otimes i d: \mathcal{K}_{p, p_{1}}\left(w_{\lambda} ; X\right) \rightarrow L_{p}\left(\mathcal{M} ; \mathcal{K}_{p, p_{2}}\left(w_{\lambda}^{\alpha}\right)\right)
$$

is completely bounded and hence $(T \otimes i d) u_{\theta, \lambda}$ is completely bounded. Thus, we derive that $\left(u_{\eta, \mu} \otimes i d_{L_{p}(\mathcal{M})}\right)(T \otimes i d)$ is completely bounded. Since $u_{\eta, \mu} \otimes i d_{L_{p}(\mathcal{M})}$ is a cb-embedding by Proposition 2.5. we obtain

$$
\left\|T \otimes i d: S_{q}(X) \rightarrow L_{p}\left(\mathcal{M} ; S_{q}\right)\right\|_{c b} \leq c\left(p, q, p_{1}, p_{2}\right) k_{\left(p_{1}, p_{2}\right)}(X) .
$$

Remark 2.10. Keeping track of constants, we have

$$
\begin{aligned}
c\left(p, q, p_{1}, p_{2}\right) & \lesssim \frac{p p_{2}}{p p_{2}+p-p_{2}} \inf _{\lambda>1} \frac{c_{2}(\lambda, \theta)^{2}}{c_{1}\left(\lambda^{\alpha}, \theta / \alpha\right)^{2}} \\
& =\frac{p p_{2}}{p p_{2}+p-p_{2}} \inf _{\lambda>1} \frac{\left(2 \lambda-\lambda^{\theta}-\lambda^{1-\theta}\right)\left(\lambda^{\alpha-\theta}-1\right)}{\left(\lambda^{1-\theta}-1\right)\left(\lambda^{\theta}+\lambda^{\alpha-\theta}-2\right)}
\end{aligned}
$$




$$
\leq \frac{p p_{2}}{p p_{2}+p-p_{2}} \lim _{\lambda \rightarrow 1^{+}} \frac{2 \lambda-\lambda^{\theta}-\lambda^{1-\theta}}{\lambda^{1-\theta}-1}=\frac{p p_{2}}{p p_{2}+p-p_{2}} \frac{1}{1-\theta},
$$

unless $\left(p, p_{2}\right)=(1, \infty)$, in which case the first term on the right behaves like 1 .

\section{MAUREY's FACTORIZATION AND APPliCATIONS}

We now prove an operator space form of Maurey's factorization theorem. Then we will establish some selected applications for operator spaces, noncommutative $L_{p}$ spaces and Fourier multipliers.

3.1. Maurey's factorization. Let us begin with some basic inequalities to be used below. We refer the reader to the Introduction for the definition of the operator space analogs of cotype $p$ and absolutely $(p, 1)$-summing maps.

Lemma 3.1. Let $2 \leq p \leq \infty$ :

i) If $T$ has cb-cotype $p$, then

$$
\pi_{p, 1}^{c b}(T) \leq c_{p}^{c b}(T) .
$$

ii) $i d_{L_{p}(\mathcal{M})}$ is completely $(p, 1)$-summing for any algebra $\mathcal{M}$.

iii) Let us consider two von Neumann algebras $\mathcal{M}, \mathcal{N}$ and assume that the map $T: L_{q}(\mathcal{M}) \rightarrow L_{p}(\mathcal{N})$ is a completely bounded map. Then, the following inequality holds for $1 \leq q \leq \infty$

$$
\left\|T \otimes i d: L_{q}\left(\mathcal{M} ; \ell_{1}\right) \rightarrow \ell_{p}\left(L_{p}(\mathcal{N})\right)\right\|_{c b} \leq\|T\|_{c b} .
$$

Proof. Consider $\Omega=\mathbb{T}^{\mathbb{N}}$ equipped with the product topology and the corresponding Haar measure $\mu$. Clearly, the map $j: \ell_{1} \rightarrow C(\Omega)$ given by $j(\alpha)(\omega)=\sum_{k} \omega_{k} \alpha_{k}$ is a complete contraction. Hence, we have

$$
\left\|j \otimes i d_{X}: \ell_{1} \otimes_{\min } X \rightarrow L_{\infty}(\Omega) \otimes_{\min } X\right\|_{c b} \leq 1 .
$$

The inclusion $L_{\infty}(\Omega ; X) \subset L_{p}(\Omega ; X)$ is also completely contractive and

$$
j \otimes i d_{X}\left(\ell_{1} \otimes_{\min } X\right) \subset \operatorname{Rad}_{p}(X) .
$$

Hence i) follows by definition. To prove ii) it suffices to show that the space $L_{p}(\mathcal{M})$ has cb-cotype $p$. Let $\Lambda: f \in L_{\infty}(\Omega) \bar{\otimes} \mathcal{M} \mapsto\left(\int_{\Omega} f \varepsilon_{k} d \mu\right)_{k \geq 1} \in \ell_{\infty}(\mathcal{M})$ be the Rademacher coefficient map. $\Lambda$ is a complete contraction and coincides with the orthogonal projection $\Lambda: L_{2}\left(\Omega ; L_{2}(\mathcal{M})\right) \rightarrow \ell_{2}\left(L_{2}(\mathcal{M})\right)$. Thus, by interpolation we deduce that $\Lambda: L_{p}\left(\Omega ; L_{p}(\mathcal{M})\right) \rightarrow \ell_{p}\left(L_{p}(\mathcal{M})\right)$ is a contraction. We conclude by restriction to $\operatorname{Rad}_{p}\left(L_{p}(\mathcal{M})\right)$. Assertion iii) now follows from the fact that the inclusion $L_{q}\left(\mathcal{M} ; \ell_{1}\right) \subset L_{q}(\mathcal{M}) \otimes_{\min } C(\Omega)$ is completely contractive. Indeed, in that case, we may compose with

$$
\begin{aligned}
L_{q}(\mathcal{M}) \otimes_{\min } C(\Omega) & \stackrel{T}{\longrightarrow} L_{p}(\mathcal{N}) \otimes_{\min } C(\Omega) \\
& \stackrel{i d}{\longrightarrow} L_{p}\left(\Omega ; L_{p}(\mathcal{N})\right) \\
& \stackrel{\Lambda}{\longrightarrow} \ell_{p}\left(L_{p}(\mathcal{N})\right) .
\end{aligned}
$$

It therefore suffices to show that for every $\omega \in \Omega$, the map

$$
\phi_{\omega}: L_{q}\left(\mathcal{M} ; \ell_{1}\right) \rightarrow L_{q}(\mathcal{M}) \quad \text { with } \quad \phi_{\omega}(x)=\sum_{k} \omega_{k} x_{k}
$$


is completely contractive. Recall that $S_{q}^{m}\left(L_{q}\left(\mathcal{M} ; \ell_{1}\right)\right)=L_{q}\left(M_{m} \otimes \mathcal{M} ; \ell_{1}\right)$ and hence we just need to show that $\phi_{w}$ is a contraction for all $w \in \Omega$. Assume $x_{k}=\sum_{j} a_{k j} b_{k j}$ such that

$$
\left\|\left(\sum_{k, j} a_{k j} a_{k j}^{*}\right)^{\frac{1}{2}}\right\|_{2 q}\left\|\left(\sum_{k, j} b_{k j}^{*} b_{k j}\right)^{\frac{1}{2}}\right\|_{2 q} \leq 1 .
$$

Then, the Cauchy-Schwartz inequality implies

$$
\begin{aligned}
\left\|\sum_{k} \omega_{k} x_{k}\right\|_{q} & =\left\|\sum_{k, j} \omega_{k} a_{k j} b_{k j}\right\|_{q} \\
& \leq\left\|\left(\sum_{k, j} a_{k j} a_{k j}^{*}\right)^{\frac{1}{2}}\right\|_{2 q}\left\|\left(\sum_{k, j}\left|\omega_{k}\right|^{2} b_{k j}^{*} b_{k j}\right)^{\frac{1}{2}}\right\|_{2 q} \leq 1 .
\end{aligned}
$$

Lemma 3.2. Let $1 \leq p<\infty$. Then $L_{p}(\mathcal{M})$ has cb-cotype $q=\max \left\{p, p^{\prime}\right\}$.

Proof. Let $\Omega=\{-1,1\}^{n}$ with Haar measure $\mu$. Given $2 \leq p \leq \infty$ and arguing as above, we know that the map $\Lambda: L_{p}\left(\Omega, L_{p}(\mathcal{M})\right) \rightarrow \ell_{p}\left(L_{p}(\mathcal{M})\right)$ defined by $\Lambda(f)=\left(\int f \varepsilon_{k} d \mu\right)_{k \leq n}$ is a complete contraction. This yields the result for $p \geq 2$. When $p<2$ we note that $\Lambda: L_{\infty}\left(\Omega ; L_{1}(\mathcal{M})\right) \rightarrow \ell_{\infty}\left(L_{1}(\mathcal{M})\right)$ is completely bounded. Again interpolation yields the result. The sharpness of this result is justified in Remark 3.12 below.

Lemma 3.3. Let $A$ be a $C^{*}$-algebra and $\phi$ be a state on $\mathcal{N}=A^{* *}$ whose restriction to $A$ is faithful. Let $d \in L_{1}(\mathcal{N})$ be the associated density with support e in $\mathcal{N}$. Let us set $\mathcal{N}_{e}=e \mathcal{N} e$. Then, we have

$$
\left[A, L_{1}\left(\mathcal{N}_{e}\right)\right]_{\frac{1}{p}}=L_{p}\left(\mathcal{N}_{e}\right)
$$

Proof. Following Kosaki's work, we have symmetric injective embedding of $\mathcal{N}_{e}$ in $L_{1}\left(\mathcal{N}_{e}\right) \cong\left(\mathcal{N}_{e}\right)^{*}$ given by $\iota(x)=d^{1 / 2} x d^{1 / 2}$. Let $x \in A$ and $y$ be an analytic element in $\mathcal{N}_{e}$. Then we observe that

$$
\langle\iota(x), y\rangle=\operatorname{tr}\left(d^{1 / 2} x d^{1 / 2} y\right)=\operatorname{tr}\left(d x d^{1 / 2} y d^{-1 / 2}\right)=\phi\left(x \sigma_{i / 2}(y)\right) .
$$

Since the elements of the form $\sigma_{i / 2}(y), y$ analytic, are in dense in $\mathcal{N}_{e}$, we deduce from $\iota(x)=0$ that $\phi\left(x^{*} x\right)=0$. However, $\phi$ is faithful and hence $\left(A, L_{1}\left(\mathcal{N}_{e}\right)\right)$ is indeed an interpolation couple and we may define $X_{p}=\left[A, L_{1}\left(\mathcal{N}_{e}\right)\right]_{1 / p}$. By Kaplansky's density theorem we known that $e A e$ is strongly dense in $\mathcal{N}_{e}$ and hence $d^{1 / 2} A d^{1 / 2}$ norm dense in $L_{1}\left(\mathcal{N}_{e}\right)$. Thus the interpolation couple has dense intersection. The unit ball in $X_{p}^{*}$ is the closure in the sum topology of the unit ball in

$$
Z_{p}=\left[A^{*}, L_{1}\left(\mathcal{N}_{e}\right)^{*}\right]_{\frac{1}{p}}=\left[\mathcal{N}_{e}, L_{1}\left(\mathcal{N}_{e}\right)\right]_{1-\frac{1}{p}},
$$

see 1 for further details. Here the natural inclusion map is again given by

$$
\left.n \in \mathcal{N}_{e} \mapsto d^{\frac{1}{2}} n d^{\frac{1}{2}} \in L_{1}\left(\mathcal{N}_{e}\right) \mapsto d^{\frac{1}{2}} n d^{\frac{1}{2}}\right|_{A} \in A^{*},
$$

because $A$ is the intersection in the interpolation couple. Certainly, $L_{1}\left(\mathcal{N}_{e}\right)$ is faithfully embedded in $A^{*}$. Thus in the dual picture we find exactly the symmetric version of Kosaki's embedding [25, $Z_{p}=L_{p^{\prime}}\left(\mathcal{N}_{e}\right)$. Since $L_{p^{\prime}}\left(\mathcal{N}_{e}\right)$ is reflexive, its unit ball is already closed in the sum topology. Indeed, given any converging sequence in the sum topology, it is easily checked that the limit is a cluster point of the sequence in the weak* topology. This gives $X_{p}^{*}=L_{p^{\prime}}\left(\mathcal{N}_{e}\right)$, so that the inclusion $X_{p} \subset L_{p}\left(\mathcal{N}_{e}\right)$ is isometric. The assertion then follows from the fact that the norm dense subspace $d^{1 / 2 p} A d^{1 / 2 p}$ of $L_{p}\left(\mathcal{N}_{e}\right)$ is contained in $X_{p}$. 
Lemma 3.4. Let $\mathcal{U}$ be an ultrafilter on an index set $I$ and

$$
\left(d_{i}\right)^{\bullet} \in \prod_{\mathcal{U}} L_{1}(\mathcal{M}) .
$$

Let $\phi(x)=\lim _{i, \mathcal{U}} \operatorname{tr}\left(d_{i} x\right)$ be the corresponding weak limit state and $d \in L_{1}\left(\mathcal{M}^{* *}\right)$ the corresponding nonfaithful density supported by e in $\mathcal{M}^{* *}$. Then, there exists a completely contractive map densely defined on $d^{1 / 2 p} \mathcal{M} d^{1 / 2 p}$ by

$$
u_{p}: d^{\frac{1}{2 p}} x d^{\frac{1}{2 p}} \in L_{p}\left(e \mathcal{M}^{* *} e\right) \mapsto\left(d_{i}^{\frac{1}{2 p}} x d_{i}^{\frac{1}{2 p}}\right) \bullet \prod_{\mathcal{U}} L_{p}(\mathcal{M}) .
$$

Proof. Let $e_{\mathcal{U}}$ be the support of

$$
\phi_{\mathcal{U}}\left(x_{i}\right)^{\bullet}=\lim _{i, \mathcal{U}} \operatorname{tr}\left(d_{i} x_{i}\right)
$$

and consider the $\sigma$-finite von Neumann algebra $\mathcal{M}_{\mathcal{U}}=e_{\mathcal{U}}\left[\prod_{\mathcal{U}} L_{1}(\mathcal{M})\right]^{*} e_{\mathcal{U}}$. The image of $u_{p}$ sits on $L_{p}\left(\mathcal{M}_{\mathcal{U}}\right)$ and $\phi_{\mathcal{U}}$ is faithful on $\mathcal{M}_{\mathcal{U}}$. Hence, the spaces $L_{p}\left(\mathcal{M}_{\mathcal{U}}\right)$ interpolate by Kosaki's result. Let $f$ be the support of $\phi$ in $\mathcal{M}$ and $e$ be the support of $\phi$ in $\mathcal{M}^{* *}$. Note that $e \leq f$. We apply Lemma 3.3 to $A=\mathcal{M}_{f}=f \mathcal{M} f$ and obtain

$$
L_{p}\left(e \mathcal{M}^{* *} e\right)=\left[\mathcal{M}_{f}, L_{1}\left(e \mathcal{M}^{* *} e\right)\right]_{\frac{1}{p}} .
$$

Therefore, the map $u_{p}$ is obtained by interpolation. Clearly $u_{\infty}(x)=e_{\mathcal{U}}(x)^{\bullet} e_{\mathcal{U}}$ is a complete contraction. The interesting part is the case $p=1$. For a positive $x \in \mathcal{M}$, we note that

$$
\left\|\left(d_{i}^{\frac{1}{2}} x d_{i}^{\frac{1}{2}}\right)^{\bullet}\right\|_{\prod_{\mathcal{U}} L_{1}(\mathcal{M})}=\lim _{i, \mathcal{U}} \operatorname{tr}\left(d_{i}^{\frac{1}{2}} x d_{i}^{\frac{1}{2}}\right)=\lim _{i, \mathcal{U}} \operatorname{tr}\left(d_{i} x\right)=\phi(x) .
$$

For a positive element $x \in \mathcal{M}^{* *}$, we may apply Kaplansky's density theorem and approximate $x^{1 / 2}$ in SOT $\cap \mathrm{SOT}^{*}$ by a net $x_{\lambda} \in \mathcal{M}$ such that $\left\|x_{\lambda}\right\| \leq\left\|x^{1 / 2}\right\|$. Then we have

$$
\lim _{i, \mathcal{U}}\left\|\left(x_{\lambda}-x_{\mu}\right) d_{i}^{\frac{1}{2}}\right\|_{2}^{2}=\lim _{i, \mathcal{U}} \operatorname{tr}\left(d_{i}\left|x_{\lambda}-x_{\mu}\right|^{2}\right)=\phi\left(\left|x_{\lambda}-x_{\mu}\right|^{2}\right) .
$$

Hence, $\left(x_{\lambda} d_{i}^{\frac{1}{2}}\right)^{\bullet}$ is Cauchy in $\prod_{\mathcal{U}} L_{2}(\mathcal{M})$ with limit $\left(x^{\frac{1}{2}} d_{i}^{\frac{1}{2}}\right)^{\bullet}$ because

$$
\begin{aligned}
\left(\phi\left(\left|x_{\lambda}-x_{\mu}\right|^{2}\right)\right)^{1 / 2} & \leq \phi\left(\left|x_{\lambda}-x\right|^{2}\right)^{\frac{1}{2}}+\phi\left(\left|x_{\mu}-x\right|^{2}\right)^{\frac{1}{2}} \\
& =\left(\phi\left(x_{\lambda}^{*} x_{\lambda}\right)+\phi\left(x^{*} x\right)-\phi\left(x_{\lambda}^{*} x\right)-\phi\left(x^{*} x_{\lambda}\right)\right)^{\frac{1}{2}} \\
& +\left(\phi\left(x_{\mu}^{*} x_{\mu}\right)+\phi\left(x^{*} x\right)-\phi\left(x_{\mu}^{*} x\right)-\phi\left(x^{*} x_{\mu}\right)\right)^{\frac{1}{2}}
\end{aligned}
$$

converges to 0 . Moreover, we have

$$
u_{1}(x)=\left(d_{i} x^{\frac{1}{2}}\right)^{\bullet}\left(x^{\frac{1}{2}} d_{i}\right)^{\bullet} \in \prod_{\mathcal{U}} L_{1}(\mathcal{M}) .
$$

Now let $x \in e \mathcal{M}^{* *} e$ be a self-adjoint element. Then we recall from [7] that

$$
\lim _{i, \mathcal{U}}\left\|d_{i}^{\frac{1}{2}} x d_{i}^{\frac{1}{2}}\right\|_{1} \leq \inf _{x=x_{1}-x_{2}} \phi\left(x_{1}\right)+\phi\left(x_{2}\right)=\left\|d^{\frac{1}{2}} x d^{\frac{1}{2}}\right\|_{1}
$$

where the infimum is taken over positive elements in $e \mathcal{M}^{* *} e$. This implies that

$$
u_{1}: d^{\frac{1}{2}} x d^{\frac{1}{2}} \in L_{1}\left(e \mathcal{M}^{* *} e\right) \rightarrow\left(d_{i}^{\frac{1}{2}} x d_{i}^{\frac{1}{2}}\right)^{\bullet} \in \prod_{\mathcal{U}} L_{1}(\mathcal{M}),
$$

is a c.p. map with $u_{1}^{*}(\mathbf{1})=\mathbf{1}$. Hence, $u_{1}$ and $u_{1}^{*}$ are contractions. Interpolation and the density of $\left[\mathcal{M}_{f}, L_{1}\left(e \mathcal{M}^{* *} e\right)\right]_{1 / p} \subset L_{p}\left(e \mathcal{M}^{* *} e\right)$ implies the result. Since the same argument holds for $M_{m}\left(e \mathcal{M}^{* *} e\right), u_{p}$ is a complete contraction. 
Proof of Theorem A. Let us begin by proving the statement i). Let $\mathcal{N}=A^{* *}$ and consider the adjoint mapping $T^{*}: X^{*} \rightarrow A^{*}$. Since $A^{*} \simeq_{c b} L_{1}\left(\mathcal{N}^{\mathrm{op}}\right)$ and $T$ is a completely $(p, 1)$-summing map we deduce from (the dual version of) Remark 1.4 that $\left\|T^{*} \otimes i d: \ell_{p^{\prime}}\left(X^{*}\right) \rightarrow L_{1}\left(\mathcal{N}^{\mathrm{op}} ; \ell_{\infty}\right)\right\|_{c b} \leq \pi_{p, 1}^{c b}(T)$. According to Theorem $\mathrm{B}$, this implies

$$
\left\|T^{*} \otimes i d: S_{q^{\prime}}\left(X^{*}\right) \rightarrow L_{1}\left(\mathcal{N}^{\mathrm{op}} ; S_{q^{\prime}}\right)\right\|_{c b} \leq c(p, q) \pi_{p, 1}^{c b}(T) .
$$

Dualizing again, we obtain the following key inequality

$$
\left\|T \otimes i d: A\left(S_{q}\right) \rightarrow S_{q}(X)\right\|_{c b} \leq c(p, q) \pi_{p, 1}^{c b}(T) .
$$

Here we interpret $A\left(S_{q}\right)$ as in Remark 1.4

$$
\begin{aligned}
A\left(S_{q}^{m}\right) & =\left[M_{m}(A), A\left(S_{1}^{m}\right)\right]_{\frac{1}{q}} \\
& \subset\left[L_{\infty}\left(A^{* *} ; S_{\infty}^{m}\right), L_{\infty}\left(A^{* *} ; S_{1}^{m}\right)\right]_{\frac{1}{q}}=L_{\infty}\left(\mathcal{N} ; S_{q}^{m}\right) .
\end{aligned}
$$

Now we follow Pisier and apply the Grothendieck-Pietsch separation argument as in [39, Theorem 5.1]. Namely, the substitute of the auxiliary Theorem 5.3 there for $A \otimes_{\min } S_{q}$ has to be replaced here by the fact that $A\left(S_{q}\right) \subset L_{\infty}\left(\mathcal{N} ; S_{q}\right)$ is understood as a conditional $L_{\infty}$ space with norm given by

$$
\|x\|_{A\left(S_{q}\right)}=\sup \left\{\|\alpha x \beta\|_{L_{q}\left(\mathcal{N} \bar{\otimes} \mathcal{B}\left(\ell_{2}\right)\right)} \mid\|\alpha\|_{L_{2 q}(\mathcal{N})},\|\beta\|_{L_{2 q}(\mathcal{N})} \leq 1\right\} .
$$

Then, it turns out that Pisier's argument in [39] generalizes verbatim to this setting and we find nets $\left(a_{\lambda}\right)$ and $\left(b_{\lambda}\right)$ in the positive part of the unit ball of $L_{2 q}(\mathcal{N})$ satisfying the inequality

$$
\|T(x)\|_{S_{q}(X)} \leq c(p, q) \pi_{p, 1}^{c b}(T) \lim _{\lambda}\left\|a_{\lambda} x b_{\lambda}\right\|_{S_{q}\left(L_{q}(\mathcal{N})\right)} .
$$

On $M_{2}(\mathcal{N})$, we define the state

$$
\phi(x)=\lim _{\lambda} \frac{1}{2}\left[\operatorname{tr}\left(a_{\lambda}^{2 q} x_{11}\right)+\operatorname{tr}\left(b_{\lambda}^{2 q} x_{22}\right)\right] .
$$

Let $d \in L_{1}\left(M_{2}(\mathcal{N})\right)$ be the density of $\phi$. We also use the notation $d_{a}, d_{b}$ for the densities of the states $\phi_{a}(x)=\lim _{\lambda} \operatorname{tr}\left(a_{\lambda}^{2 q} x\right)$ and $\phi_{b}(x)=\lim _{\lambda} \operatorname{tr}\left(b_{\lambda}^{2 q} x\right)$. According to Lemma 3.4, we see that

$$
u_{q}\left(d^{\frac{1}{2 q}} x d^{\frac{1}{2 q}}\right)=\left(d_{\lambda}^{\frac{1}{2 q}} x d_{\lambda}^{\frac{1}{2 q}}\right)^{\bullet} \quad \text { with } \quad d_{\lambda}=\frac{1}{2}\left(e_{11} \otimes a_{\lambda}^{2 q}+e_{22} \otimes b_{\lambda}^{2 q}\right) .
$$

is a complete contraction. Restricting this to the $(1,2)$ entry, we deduce that

$$
\begin{aligned}
\lim _{\lambda} 2^{-\frac{1}{q}}\left\|a_{\lambda} x b_{\lambda}\right\|_{q} & =\lim _{\lambda}\left\|d_{\lambda}^{\frac{1}{2 q}}\left(e_{12} \otimes x\right) d_{\lambda}^{\frac{1}{2 q}}\right\|_{q} \\
& \leq\left\|d^{\frac{1}{2 q}}\left(e_{12} \otimes x\right) d^{\frac{1}{2 q}}\right\|_{q}=2^{-\frac{1}{q}}\left\|d_{a}^{\frac{1}{2 q}} x d_{b}^{\frac{1}{2 q}}\right\|_{q} .
\end{aligned}
$$

Moreover, the same chain of inequalities holds for $x$ replaced by an element in $M_{m}(A)$. The first assertion then follows immediately. Indeed, it just remains to choose the densities $\delta_{1}^{2 q}=d_{a}, \delta_{2}^{2 q}=d_{b}$ and define the map

$$
w\left(\delta_{1} x \delta_{2}\right)=T(x) .
$$

To prove ii), we follow an argument by Haagerup. According to the first part applied to $A=\mathcal{M}$, we find $\delta_{1}, \delta_{2} \in L_{2 q}^{+}\left(\mathcal{M}^{* *}\right)$ of norm 1 . Moreover, using the existence of a central projection $z$ in $\mathcal{M}^{* *}$ such that $\mathcal{M}=z \mathcal{M}^{* *}$, we define $d_{1}=z \delta_{1}$ and $d_{2}=z \delta_{2}$. Let $\left(z_{\lambda}\right) \subset \mathcal{M}$ be a net of contractions which converges strongly to $z$ in $\mathcal{M}^{* *}$. Then $d_{1}=\lim _{\lambda} z_{\lambda} \delta_{1}$ and $d_{2}=\lim _{\lambda} z_{\lambda} \delta_{2}$. On the other hand $\mathbf{1}_{\mathcal{M}}-z_{\lambda}$ 
converges strongly to 0 , where strongly refers this time to $\mathcal{M}$. Since $T$ is supposed to be normal, we have $T^{*}\left(X^{*}\right) \subset L_{1}(\mathcal{M})$. This implies

$$
\lim _{\lambda, \mu}\left\langle x^{*}, T\left(z_{\lambda} x z_{\mu}\right)\right\rangle=\lim _{\lambda, \mu}\left\langle z_{\lambda} T^{*}\left(x^{*}\right) z_{\mu}, x\right\rangle=\left\langle x^{*}, T(x)\right\rangle .
$$

Let $x \in S_{q}(\mathcal{M})$ and $x^{*}$ in the unit ball of $S_{q^{\prime}}\left(X^{*}\right)$ so that

$$
\|T(x)\|_{S_{q}(X)}=\left|\left\langle x^{*}, T(x)\right\rangle\right| .
$$

Then we find

$$
\begin{aligned}
\|T(x)\|_{S_{q}(X)} & =\lim _{\lambda, \mu}\left|\left\langle x^{*}, T\left(z_{\lambda} x z_{\mu}\right)\right\rangle\right| \\
& \leq c(p, q) \pi_{p, 1}^{c b}(T) \lim _{\lambda, \mu}\left\|\delta_{1} z_{\lambda} x z_{\mu} \delta_{2}\right\|_{S_{q}\left(L_{q}\left(\mathcal{M}^{* *}\right)\right)} \\
& =c(p, q) \pi_{p, 1}^{c b}(T)\left\|d_{1} x d_{2}\right\|_{S_{q}\left(L_{q}\left(\mathcal{M}^{* *}\right)\right)} .
\end{aligned}
$$

This shows that $v\left(d_{1} x d_{2}\right)=T(x)$ is continuous and even completely bounded. The proof of iii) follows the same pattern above. We first dualize and consider the map $T^{*}: X^{*} \rightarrow L_{s^{\prime}}(\mathcal{M})$, which is $\left(p^{\prime}, \infty\right)$-convex. Indeed, this follows by duality since

$$
L_{s}\left(\mathcal{M} ; \ell_{1}\right) \stackrel{i d}{\longrightarrow} \ell_{1} \otimes_{\min } L_{s}(\mathcal{M}) \stackrel{T}{\longrightarrow} \ell_{p}(X)
$$

is completely bounded. Then, since $s^{\prime}<q^{\prime}<p^{\prime} \wedge \infty$, we may apply Theorem B to deduce that $T^{*} \otimes i d: S_{q^{\prime}}\left(X^{*}\right) \rightarrow L_{s^{\prime}}\left(\mathcal{M} ; S_{q^{\prime}}\right)$ is completely bounded with cb-norm controlled by $c(p, q, s) \pi_{p, 1}^{c b}(T)$. Dualizing back and with the help of the Grothendieck-Pietsch factorization theorem (adapted to this setting as indicated above), we find nets $\left(a_{\lambda}\right),\left(b_{\lambda}\right)$ in the positive part of the unit ball of $L_{2 w}(\mathcal{M})$ such that

$$
\|T(x)\|_{S_{q}(X)} \leq c(p, q, s) \pi_{p, 1}^{c b}(T) \lim _{\lambda}\left\|a_{\lambda} x b_{\lambda}\right\|_{S_{q}\left(L_{q}(\mathcal{M})\right)} .
$$

Let us assume for simplicity that $a_{\lambda}=b_{\lambda}=d_{\lambda}$. Recall that this can always be done using the $2 \times 2$ matrix trick from above. Then we define the following weak* limit in $L_{s^{\prime}}(\mathcal{M})$

$$
\operatorname{tr}(d x)=\lim _{\lambda} \operatorname{tr}\left(d_{\lambda}^{2 w / s^{\prime}} x\right) .
$$

The assertion is obtained from the inequality

$$
\lim _{\lambda}\left\|d_{\lambda} x d_{\lambda}\right\|_{q} \leq\left\|d^{s^{\prime} / 2 w} x d^{s^{\prime} / 2 w}\right\|_{q}
$$

which follows by approximating $x \sim d_{\lambda}^{w / s} z d_{\lambda}^{w / s}$ and applying Lemma 3.4

Remark 3.5. According to Remark 2.10, we obtain

$$
c(p, q) \lesssim \frac{1}{1-\frac{p}{q}}
$$

We also have the weaker estimate $c(p, q, s) \lesssim q(s-p) /(q-p)$ for $s<\infty$.

Remark 3.6. We may define canonically

$$
\pi_{p, q}^{c b}(T)=\left\|i d \otimes T: \ell_{q} \otimes_{\min } X \rightarrow \ell_{p}(Y)\right\|_{c b}
$$

as the completely $(p, q)$-summing norm of $T: X \rightarrow Y$. At the time of this writing, it is not clear whether $\pi_{p, p}^{c b}(T)=\pi_{p}^{o}(T)$ holds for all maps $T$. However, Pisier's factorization theorem immediately implies that every completely $p$-summing map is completely $(p, p)$ summing, and $\pi_{p, p}^{c b}(T) \leq \pi_{p}^{o}(T)$. If in addition $T$ is a normal 
map on an injective von Neumann algebra, then the norms are equivalent. Indeed, let $T^{*}: Y^{*} \rightarrow L_{1}(\mathcal{M})$ be the adjoint, $\mathcal{M}$ injective such that

$$
\pi_{p, p}^{c b}(T)=\left\|i d \otimes T^{*}: \ell_{p^{\prime}}\left(Y^{*}\right) \rightarrow L_{1}\left(\mathcal{M} ; \ell_{p^{\prime}}\right)\right\|_{c b}<\infty .
$$

Recall from [15] that we have a cb-embedding $j: S_{p^{\prime}}^{m} \rightarrow L_{1}\left(\mathcal{N} ; \ell_{p^{\prime}}\right)$, so that

$$
i d \otimes j: L_{1}\left(\mathcal{M} ; S_{p^{\prime}}^{m}\right) \rightarrow L_{1}\left(\mathcal{M} \bar{\otimes} \mathcal{N} ; \ell_{p^{\prime}}\right)
$$

is an isomorphic embedding. This map uses independent copies and hence it is easy to check that $j \otimes i d_{Y^{*}}: S_{p^{\prime}}^{m}\left(Y^{*}\right) \rightarrow L_{1}\left(\mathcal{N} ; \ell_{p^{\prime}}\left(Y^{*}\right)\right)$ remains bounded with a constant $c(p)$. Then we find the following diagram

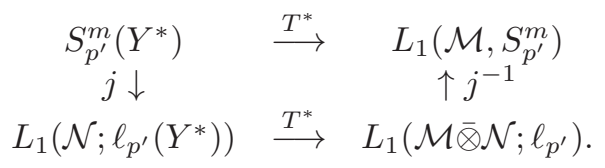

The two maps $\downarrow$ and $\uparrow$ are bounded, and hence

$$
\left\|i d_{S_{p^{\prime}}^{m}} \otimes T^{*}: S_{p^{\prime}}^{m}\left(Y^{*}\right) \rightarrow L_{1}\left(\mathcal{M} ; S_{p^{\prime}}^{m}\right)\right\| \leq c(p) \pi_{p, p}^{c b}(u)
$$

is still bounded with constants independent of $m$. This completes the argument.

3.2. Applications I. Operator spaces. Our first application is an operator space analog of Rosenthal's theorem [43] for subspaces of (commutative or not) $L_{p}$ spaces. This partly justifies our definition of cb-cotype, see [5, 15, 27, 32] for related notions.

Proof of Corollary A1. We shall prove i) $\Rightarrow$ ii $) \Rightarrow$ iii $\Rightarrow$ i). The first implication follows from Lemma 3.1 For the second implication, assume that $X^{*}$ is completely $\left(p_{0}^{\prime}, 1\right)$-summing for some index $p<p_{0}<2$ and let $j: X \rightarrow L_{p}(\mathcal{M})$ be the inclusion map. Take the (necessarily normal) adjoint map $T=j^{*}: L_{p^{\prime}}(\mathcal{M}) \rightarrow X^{*}$. Given $p_{0}^{\prime}<q^{\prime}<p^{\prime}$, the map $T: \ell_{1} \otimes_{\min } L_{p^{\prime}}(\mathcal{M}) \rightarrow \ell_{p_{0}^{\prime}}\left(X^{*}\right)$ is completely bounded since $i d_{X^{*}}$ is completely $\left(p_{0}^{\prime}, 1\right)$-summing and

$$
\ell_{1} \otimes_{\min } L_{p^{\prime}}(\mathcal{M}) \stackrel{T}{\longrightarrow} \ell_{1} \otimes_{\min } X^{*} \stackrel{i d}{\longrightarrow} \ell_{p_{0}^{\prime}}\left(X^{*}\right) .
$$

In particular, $T$ satisfies the assertion of Theorem A. Let $v: L_{q^{\prime}}(\mathcal{M}) \rightarrow X^{*}$ be the corresponding map. Then $v^{*}: X \rightarrow L_{q}(\mathcal{M})$ is also completely bounded and $d_{1} v^{*}(x) d_{2}=j(x)$. In particular, since $d_{1}, d_{2}$ are norm 1 in $L_{2 w}(\mathcal{M})$ and $\frac{1}{p}=\frac{1}{q}+\frac{1}{w}$

$$
\|x\|_{M_{m}(X)}=\|j(x)\|_{M_{m}\left(L_{p}(\mathcal{M})\right)}=\left\|d_{1} v^{*}(x) d_{2}\right\|_{M_{m}\left(L_{p}(\mathcal{M})\right)} \leq\left\|v^{*}(x)\right\|_{M_{m}\left(L_{q}(\mathcal{M})\right)} .
$$

Thus, $X$ is cb-isomorphic to $v^{*}(X) \subset L_{q}(\mathcal{M})$. For the third implication, the Rademacher transform map $\Lambda: f \in \operatorname{Rad}\left(L_{q^{\prime}}(\mathcal{M})\right) \mapsto\left(\int_{\Omega} f \varepsilon_{k} d \mu\right) \in \ell_{q^{\prime}}\left(L_{q^{\prime}}(\mathcal{M})\right)$ is completely contractive and this remains true for every quotient of $L_{q^{\prime}}(\mathcal{M})$. In particular, $X^{*}$ has cb-cotype $q^{\prime}$. The proof is complete.

Corollary 3.7. If $p \geq 2$ and $i d_{X}$ is completely $(p, 1)$-summing

$$
\Pi_{1}^{o}(X, Y)=\Pi_{q^{\prime}}^{o}(X, Y) \text { for all operator spaces } Y \text { and } q>p .
$$

Proof. The inclusion

$$
\Pi_{1}^{o}(X, Y) \subset \Pi_{q^{\prime}}^{o}(X, Y)
$$

is well-known. For the converse, we consider $u: M_{m} \rightarrow X$ and note that

$$
\pi_{p, 1}^{c b}(u) \leq\|u\|_{c b} \pi_{p, 1}^{c b}\left(i d_{X}\right) .
$$


Theorem $₫$ for $\mathcal{M}=M_{m}$ gives $a, b \in S_{2 q}^{m}$ and a cb-map $w: S_{q}^{m} \rightarrow X$ such that

$$
u=w \circ M_{a b} \quad \text { and } \quad\|a\|_{2 q}\|w\|_{c b}\|b\|_{2 q} \leq c(p, q)\|u\|_{c b} \pi_{p, 1}^{c b}\left(i d_{X}\right) .
$$

The argument now follows by a standard duality argument. We refer the reader to [39, Chapter 7] for a brief review of the duality theory of $p$-summing maps both in the Banach and operator space settings. We shall also use the $p$-nuclear norm $\nu_{p}^{o}$ and the fact that it is trace dual to $\pi_{q^{\prime}}^{o}$, see [10, Chapter 3]. If $T: X \rightarrow Y$ and $v: Y \rightarrow M_{m}$, we deduce that

$$
\begin{aligned}
|\operatorname{tr}(v T u)| & =\left|\operatorname{tr}\left(M_{a b} v T w\right)\right| \\
& \leq \nu_{q}^{o}\left(M_{a b} v\right) \pi_{q^{\prime}}^{o}(T w) \\
& \leq\|v\|_{c b}\|a\|_{2 q}\|w\|_{c b}\|b\|_{2 q} \pi_{q^{\prime}}^{o}(T) \\
& \leq c(p, q) \pi_{p, 1}^{c b}\left(i d_{X}\right)\|v\|_{c b}\|u\|_{c b} \pi_{q^{\prime}}^{o}(T) .
\end{aligned}
$$

Thus we obtain the inequality

$$
\sup _{\|u\|_{c b},\|v\|_{c b} \leq 1}|\operatorname{tr}(v T u)| \leq c(p, q) \pi_{p, 1}^{c b}\left(i d_{X}\right) \pi_{q^{\prime}}^{o}(T) .
$$

Since $\mathcal{C B}\left(Y, M_{m}\right)=\left[S_{1}^{m}(Y)\right]^{*}$ and $\mathcal{C B}\left(M_{m}, X\right)=S_{1}^{m} \otimes_{\min } X$, we get

$$
\left\|T \otimes i d: S_{1}^{m} \otimes_{\min } X \rightarrow S_{1}^{m}(Y)\right\| \leq c(p, q) \pi_{p, 1}^{c b}\left(i d_{X}\right) \pi_{q^{\prime}}^{o}(T),
$$

but the left hand side is the completely 1-summing of $T$. The proof is complete.

Proof of Corollary A2. The first assertion follows from Theorem A, while the second assertion follows from Lemma 3.2 applied to $O H$ and Corollary 3.7

3.3. Applications II. Noncommutative $L_{p}$ spaces. We now investigate some further consequences of our results for linear maps between noncommutative $L_{p}$ spaces equipped with their natural operator space structures.

Corollary 3.8. Let $2 \leq q_{1}<p_{1}<q_{2} \leq p_{2} \leq \infty$. Assume that

$$
T: L_{p_{2}}(\mathcal{M}) \rightarrow L_{p_{1}}(\mathcal{M}) \quad \text { and } \quad S: L_{q_{2}}(\mathcal{N}) \rightarrow L_{q_{1}}(\mathcal{N})
$$

are completely bounded maps with $\mathcal{M}, \mathcal{N}$ being QWEP von Neumann algebras. In the case $p_{2}=\infty$ or $q_{2}=\infty$, assume in addition that the corresponding map is normal. Then, the following map is completely bounded

$$
T \otimes S: L_{p_{2}}\left(\mathcal{M} ; L_{q_{2}}(\mathcal{N})\right) \rightarrow L_{p_{1}}\left(\mathcal{M} ; L_{q_{1}}(\mathcal{N})\right) .
$$

Proof. If $2 \leq p_{1}<q_{2} \leq p_{2}$, we claim that

$$
(\widetilde{T \otimes i d})(x \otimes y)=y \otimes T(x)
$$

satisfies

$$
\left\|\widetilde{T \otimes i d}: L_{p_{2}}\left(\mathcal{M} ; L_{q_{2}}(\mathcal{N})\right) \rightarrow L_{q_{2}}\left(\mathcal{N} ; L_{p_{1}}(\mathcal{M})\right)\right\|_{c b} \leq c\left(p_{1}, q_{2}\right)\|T\|_{c b} .
$$

Indeed, if $p_{2}=q_{2}$ then $L_{p_{2}}\left(\mathcal{M} ; L_{p_{2}}(\mathcal{N})\right)=L_{p_{2}}(\mathcal{M} \bar{\otimes} \mathcal{N})=L_{p_{2}}\left(\mathcal{N} ; L_{p_{2}}(\mathcal{M})\right)$. Since $\mathcal{N}$ is QWEP, we deduce the assertion from the complete boundedness of $T$. A similar argument can be found in [13]. When $p_{2}>q_{2}$, we use that $L_{p_{1}}(\mathcal{M})$ has cb-cotype $p_{1}$ and Theorem A to factorize

$$
T=v \circ M_{a b}
$$


where $v: L_{q_{2}}(\mathcal{M}) \rightarrow L_{p_{1}}(\mathcal{M})$ is completely bounded and $M_{a b}(x)=a x b$ with $a, b$ positive norm 1 elements of $L_{2 s}(\mathcal{M})$ for $1 / q_{2}=1 / p_{2}+1 / s$. It is clear that the map

$$
M_{a b} \otimes i d: L_{p_{2}}\left(\mathcal{M} ; L_{q_{2}}(\mathcal{N})\right) \rightarrow L_{q_{2}}\left(\mathcal{M} ; L_{q_{2}}(\mathcal{N})\right)
$$

is completely contractive. Moreover, our argument for $p_{2}=q_{2}$ gives

$$
\begin{aligned}
\left\|v \otimes i d: L_{q_{2}}\left(\mathcal{M} ; L_{q_{2}}(\mathcal{N})\right) \rightarrow L_{q_{2}}\left(\mathcal{N} ; L_{p_{1}}(\mathcal{M})\right)\right\|_{c b} & \leq c\left(p_{1}, p_{2}, q_{2}\right) \pi_{p_{1}, 1}^{c b}(T) \\
& \leq c\left(p_{1}, p_{2}, q_{2}\right)\|T\|_{c b} .
\end{aligned}
$$

This proves our claim. Moreover, if

$$
2 \leq q_{1}<p_{1}<q_{2} \leq p_{2}
$$

the same argument for $\widetilde{i d \otimes S}$ yields

$$
\left\|\widetilde{i d \otimes S}: L_{q_{2}}\left(\mathcal{N} ; L_{p_{1}}(\mathcal{M})\right) \rightarrow L_{p_{1}}\left(\mathcal{M} ; L_{q_{1}}(\mathcal{N})\right)\right\|_{c b} \leq c\left(p_{1}, q_{1}, q_{2}\right)\|S\|_{c b} .
$$

Combining the two estimates, we deduce the assertion. The proof is complete.

Corollary 3.9. If $2 \leq p<q<\infty$ and $\mathcal{M}, \mathcal{N}$ are hyperfinite

$$
\mathcal{C B}\left(L_{1}(\mathcal{M}), L_{p}(\mathcal{N})\right)=\Pi_{q}^{o}\left(L_{1}(\mathcal{M}), L_{p}(\mathcal{N})\right) .
$$

Proof. Since $1<p^{\prime} \leq 2$ and according to [17, 18, we have a cb-embedding $j: L_{p^{\prime}}(\mathcal{N}) \rightarrow L_{1}(\mathcal{A})$ for some hyperfinite von Neumann algebra $\mathcal{A}$. The dual map $j^{*}: \mathcal{A}^{o p} \rightarrow L_{p}(\mathcal{N})$ is a complete surjection. Let $u: L_{1}(\mathcal{M}) \rightarrow L_{p}(\mathcal{N})$ be a completely bounded map and $u^{*}: L_{p^{\prime}}(\mathcal{N}) \rightarrow \mathcal{M}^{o p}$ its adjoint map. Since $\mathcal{M}$ is injective, we have a cb-norm preserving extension $w: L_{1}(\mathcal{A}) \rightarrow \mathcal{M}^{o p}$. The restriction $\tilde{u}$ of $w^{*}\left(\mathcal{M}^{o p}\right)^{*} \rightarrow \mathcal{A}^{o p}$ to $L_{1}(\mathcal{M})$ gives an extension of $u: L_{1}(\mathcal{M}) \rightarrow \mathcal{A}^{o p}$ such that $u=j^{*} w^{*}$ and

$$
\|\tilde{u}\|_{c b} \leq\|u\|_{c b}\|j\|_{c b}\left\|j^{-1}\right\|_{c b} \leq c\|u\|_{c b} .
$$

Since $L_{p}(\mathcal{M})$ has cb-cotype $p$ from Lemma 3.2 and $j^{*}$ is normal, we know from Theorem A that $j^{*}$ is completely $q$-summing. Recall that the fact that $\mathcal{A}$ is injective is used here to ensure that $\mathcal{A}\left(S_{q}^{m}\right)=\mathcal{A} \otimes_{\min } S_{q}^{m}$. Thus we conclude $u=j^{*} \tilde{u}$ is also completely $q$-summing.

Corollary 3.10. If $\mathcal{M}$ is finite and hyperfinite and

$$
T: L_{1}(\mathcal{M}) \rightarrow L_{2}(\mathcal{M})
$$

is completely bounded, then the eigenvalues of $T: L_{2}(\mathcal{M}) \rightarrow L_{2}(\mathcal{M})$ satisfy

$$
\left(\sum_{k}\left|\lambda_{k}(T)\right|^{2}\right)^{\frac{1}{2}} \leq\|T\|_{c b}
$$

Proof. It is well-known [10, 3.4.3.13] that

$$
\left(\sum_{k}\left|\lambda_{k}(T)\right|^{q}\right)^{\frac{1}{q}} \leq \pi_{q}^{o}(T)
$$

for $2<q<\infty$. Here $\lambda_{k}(T)$ are the eigenvalues in non-decreasing order. Let us take the opportunity to correct an oversight in the proof. In [10, p.238] it was claimed that

$$
\prod_{\mathcal{U}} S_{p} \stackrel{?}{=}\left[\prod_{\mathcal{U}} S_{\infty}, \prod_{\mathcal{U}} S_{2}\right]_{\frac{2}{p}}
$$


interpolates. However, this is not an interpolation couple. Instead, one has to use Pisier's factorization theorem and use that for a positive density $d=\left(d_{i}\right) \in \prod_{\mathcal{U}} S_{1}$ the spaces

$$
L S_{p}=\operatorname{cl}\left(\left\{\left(d_{i}^{1 / 2 p} x_{i} d_{i}^{1 / 2 p}\right)^{\bullet} \mid\left(x_{i}\right)^{\bullet}=e_{\mathcal{U}}\left(x_{i}\right)^{\bullet} e_{\mathcal{U}} \in\left(\prod_{\mathcal{U}} S_{1}\right)^{*}\right\}\right) \subset \prod_{\mathcal{U}} S_{p}
$$

form an interpolation scale, due to Kosaki's interpolation theorem. In the rest of the proof one works with these spaces. In order to push the result to $q=2$, we may apply a standard tensor trick. Let $m \in \mathbb{N}$ and $j_{m}: L_{2}\left(\mathcal{M}^{\otimes_{m}}\right) \rightarrow L_{1}\left(\mathcal{M}^{\otimes_{m}}\right)$ be the natural completely contractive inclusion map. Then we deduce from Corollary 3.9 that

$$
\begin{aligned}
\left(\sum_{k=1}^{n}\left|\lambda_{k}(T)\right|^{2}\right)^{\frac{m}{2}} & =\left(\sum_{k=1}^{n^{m}}\left|\lambda_{k}\left(T^{\otimes_{m}}\right)\right|^{2}\right)^{\frac{1}{2}} \\
& \leq n^{\frac{m}{2}-\frac{m}{q}}\left(\sum_{k=1}^{n^{m}}\left|\lambda_{k}\left(T^{\otimes_{m}}\right)\right|^{q}\right)^{\frac{1}{q}} \\
& \leq n^{\frac{m}{2}-\frac{m}{q}} \pi_{q}^{o}\left(T^{\otimes_{m}} j_{m}\right) \leq n^{\frac{m}{2}-\frac{m}{q}} c(q)\left\|T^{\otimes m}\right\|_{c b} .
\end{aligned}
$$

We now claim that $\left\|T^{\otimes m}\right\|_{c b} \leq\|T\|_{c b}^{m}$. Indeed, given

$$
T: L_{1}(\mathcal{M}) \rightarrow L_{2}(\mathcal{M}) \quad \text { and } \quad S: L_{1}(\mathcal{N}) \rightarrow L_{2}(\mathcal{N}),
$$

we observe that

$$
L_{1}(\mathcal{M} \bar{\otimes} \mathcal{N}) \stackrel{S}{\longrightarrow} L_{1}\left(\mathcal{M} ; L_{2}(\mathcal{N})\right) \longrightarrow L_{2}\left(\mathcal{N} ; L_{1}(\mathcal{M})\right) \stackrel{T}{\longrightarrow} L_{2}(\mathcal{M} \bar{\otimes} \mathcal{N})
$$

where the middle map is a complete contraction by Minkowski's inequality. Hence we have $\|T \otimes S\|_{c b} \leq\|T\|_{c b}\|S\|_{c b}$. Applying it $m-1$ times, we deduce our claim and therefore we get

$$
\left(\sum_{k=1}^{n}\left|\lambda_{k}(T)\right|^{2}\right)^{\frac{m}{2}} \leq n^{\frac{m}{2}-\frac{m}{q}} c(q)\|T\|_{c b}^{m}
$$

Thus, taking $m$-th roots and sending $(m, q) \rightarrow(\infty, 2)$, the result follows.

Corollary 3.11. Let $1<p<\infty$ and $q>p \vee p^{\prime}$. If $\mathcal{M}$ is hyperfinite and the map $T: \mathcal{M} \rightarrow \mathcal{M}$ is normal with a factorization $T=v w$, where $v: L_{p}(\mathcal{M}) \rightarrow \mathcal{M}$ and $w: \mathcal{M} \rightarrow L_{p}(\mathcal{M})$ normal, both completely bounded. Then, we have

$$
\left(\sum_{k}\left|\lambda_{k}(T)\right|^{q}\right)^{\frac{1}{q}} \leq c(p, q)\|v\|_{c b}\|w\|_{c b}
$$

Proof. When $p \geq 2$, this follows from Theorem A because $w: \mathcal{M} \rightarrow L_{p}(\mathcal{M})$ is completely $q$-summing and hence $T=v w$ is also completely $q$-summing. In the case $1<p<2$, we consider $T^{*}=w^{*} v^{*}$ and deduce from Corollary 3.9 that the map $v^{*}: L_{1}(\mathcal{M}) \rightarrow L_{p^{\prime}}(\mathcal{M})$ is completely $q$-summing. Following the eigenvalue estimates from [10, p.238] and letting $T_{*}=\left.T^{*}\right|_{L_{1}}$, we know that $T_{*}^{m}$ is compact for some $m \in \mathbb{N}$. Hence $T^{m}$ is also compact and $T$ is a Riesz operator. Recall that an operator $T: X \rightarrow X$ is Riesz if for all $\varepsilon>0$ there exist $n, m \in \mathbb{N}$ and $y_{1}, \ldots, y_{m}$ such that $T^{n}\left(B_{X}\right) \subset \bigcup_{k} y_{k}+\varepsilon B_{X}$. Fortunately, we know by a result of West which can be found in [34, 3.2.26] that for a Riesz operator the eigenvalues sequence $\left(\lambda_{k}(T)\right)$ can be arranged so that $\left(\lambda_{k}(T)\right)=\left(\lambda_{k}\left(T^{*}\right)\right)$. We also refer to [34, Section 3.2] for the definition of the eigenvalue sequence respecting the algebraic multiplicity. 
Hence our estimate of the $q$-norm of $\left(\lambda_{k}\left(T_{*}\right)\right)$ implies the same estimate for the eigenvalue sequence of $T: \mathcal{M} \rightarrow \mathcal{M}$.

Remark 3.12. Let us consider an example. Given a sequence $\left(\mu_{k}\right) \in \ell_{p}$ of positive numbers, the cb-norm of the diagonal map $\Delta_{\sqrt{\mu}}: e_{k 1} \in C \mapsto \sqrt{\mu_{k}} e_{k 1} \in C_{p}$ is given by

$$
\left\|\Delta_{\sqrt{\mu}}: C \rightarrow C_{p}\right\|_{c b}=\left(\sum_{k=1}^{\infty} \mu_{k}^{p}\right)^{\frac{1}{2 p}}=\left\|\Delta_{\sqrt{\mu}}: C_{p} \rightarrow C\right\|_{c b} .
$$

Hence, $\Delta_{\sqrt{\mu}}$ factors through $S_{p}$ and $S_{p^{\prime}}$ and therefore the best possible exponent in Corollary 3.11 is indeed $p \vee p^{\prime}$. This also shows that Lemma 3.2 can not be essentially improved, because $C_{p}=R_{p^{\prime}} \subset S_{p^{\prime}}$ is a complemented subspace and hence we can not have cotype 2 , at most cotype $p$. However, for $p=2$ we know that the exponent is not attained in general because the little Grothendieck inequality fails in this form 12. Also hyperfiniteness is necessary, because in the free group algebra $V N\left(\mathbf{F}_{\infty}\right)$ every diagonal operator $\Delta_{\mu}\left(\lambda\left(g_{k}\right)\right)=\mu_{k} \lambda\left(g_{k}\right), g_{k}$ the generators, factors completely through $L_{p}\left(V N\left(\mathbf{F}_{\infty}\right)\right)$ whenever $\Delta_{\mu}: R_{p} \cap C_{p} \rightarrow R \cap C$ is completely bounded. Note here that the span of the generators is completely complemented (see 40]) and we may therefore view these maps as defined on $V N\left(\mathbf{F}_{\infty}\right)$. That is, $\mu \in \ell_{2 p}$. Hence the eigenvalues are not in $\ell_{p}$.

3.4. Applications III. Fourier multipliers. Our last application is devoted to Fourier multipliers. Let $G$ be a discrete group and let $V N(G)$ stand for the finite von Neumann algebra generated by the left regular representation $\lambda$. Given a function $\phi: G \rightarrow \mathbb{C}$, the corresponding Fourier multiplier $\lambda(g) \mapsto \phi(g) \lambda(g)$ will be denoted by $T_{\phi}$.

Corollary 3.13. If $2 \leq p<q<\infty$ and if

$$
T_{\phi}: V N(G) \rightarrow L_{p}(V N(G))
$$

is completely bounded, then $T_{\phi}: L_{q}(V N(G)) \rightarrow L_{p}(V N(G))$ satisfies

$$
\left\|T_{\phi}: L_{q}(V N(G)) \rightarrow L_{p}(V N(G))\right\|_{c b} \leq c(p, q)\left\|T_{\phi}: V N(G) \rightarrow L_{p}(V N(G))\right\|_{c b} .
$$

Proof. The algebra $\mathbb{C}[G]$ of finite sums $\sum_{g} \alpha_{g} \lambda(g)$ is dense in $L_{p^{\prime}}(V N(G))$ and $T_{\phi}^{*}(\mathbb{C}[G]) \subset \mathbb{C}[G]$. This shows that $T_{\phi}$ is normal. Theorem $\mathrm{A}$ gives two norm 1 elements $a, b \in L_{2 q}(V N(G))$ and a cb-map $v: L_{q}(V N(G)) \rightarrow L_{p}(V N(G))$ such that $T_{\phi}(x)=v(a x b)$. Let $\pi: V N(G) \rightarrow V N(G) \bar{\otimes} V N(G)$ be the representation given by $\pi(\lambda(g))=\lambda(g) \otimes \lambda(g)$. Let us show that the map

$$
\Lambda_{a b}: x \in L_{q}(V N(G)) \mapsto(\mathbf{1} \otimes a) \pi(x)(\mathbf{1} \otimes b) \in L_{q}(V N(G) \bar{\otimes} V N(G))
$$

is completely contractive. This is obvious for $q=\infty$, while for $q=2$

$$
\begin{aligned}
\left\|\sum_{g} \alpha_{g} \lambda(g) \otimes a \lambda(g) b\right\|_{2}^{2} & =\sum_{g}\left|\alpha_{g}\right|^{2}\|a \lambda(g) b\|_{2}^{2} \\
& \leq\|a\|_{4}^{2}\|b\|_{4}^{2} \sum_{g}\left|\alpha_{g}\right|^{2} \\
& =\|a\|_{4}^{2}\|b\|_{4}^{2}\left\|\sum_{g} \alpha_{g} \lambda(g)\right\|_{2}^{2} .
\end{aligned}
$$

On the other hand, note that $i d \otimes v: L_{q}(V N(G) \bar{\otimes} V N(G)) \rightarrow L_{p}(V N(G) \bar{\otimes} V N(G))$ is completely bounded. Indeed, $i d \otimes v: L_{q}\left(L_{q}\right) \rightarrow L_{q}\left(L_{p}\right)$ is clearly completely 
bounded and the inclusion $L_{q}\left(L_{p}\right) \subset L_{p}\left(L_{p}\right)$ is completely contractive. The latter assertion follows regarding the involved spaces as conditional $L_{p}$ spaces and using interpolation. Combining this with $\Lambda_{a b}$ we find that

$$
\pi\left(T_{\phi}(\lambda(g))=\phi(g) \lambda(g) \otimes \lambda(g)=\lambda(g) \otimes v(a \lambda(g) b)=(i d \otimes v) \Lambda_{a b}(\lambda(g) \otimes \lambda(g)) .\right.
$$

Finally, we observe that $\pi: L_{p}(V N(G)) \rightarrow L_{p}(V N(G) \bar{\otimes} V N(G))$ is a completely isometric embedding. This follows from the $L_{p}$ version of Fell absorption principle 33. Therefore, we conclude that $T_{\phi}=\pi^{-1}(i d \otimes v) \Lambda_{a b}$ is completely bounded.

Acknowledgement. We would like to thank the referee for a very careful reading of the paper. His/her suggestions have led to a much more transparent presentation.

\section{REFERENCES}

1. J. Bergh and J. Löfström, Interpolation Spaces. Springer-Verlag, Berlin, 1976.

2. M.D. Choi and E.G. Effros, The completely positive lifting problem for $C^{*}$-algebras. Ann. of Math. 104 (1976), 585-609.

3. E.G. Effros and Z.J. Ruan, A new approach to operator spaces. Can. Math. Bull. 34 (1991), 329-337.

4. E.G. Effros and Z.J. Ruan, Operator Spaces. London Math. Soc. Monogr. 23, Oxford University Press, 2000.

5. J. García-Cuerva and J. Parcet, Quantized orthonormal systems: A non-commutative Kwapien theorem. Studia Math. 155 (2003), 273-294.

6. U. Haagerup, Self-polar forms, conditional expectations and the weak expectation property for $C^{*}$-algebras. Unpublished manuscript, 1995.

7. U. Haagerup, M. Junge and $\mathrm{Q}, \mathrm{Xu}, A$ reduction method for noncommutative $L_{p}$ spaces and applications. To appear in Trans. Amer. Math. Soc.

8. U. Haagerup and M. Musat, The Effros-Ruan conjecture for bilinear forms on $C^{*}$-algebras. Invent. Math. 174 (2008), 139-163.

9. A. Harcharras, On some "stability" properties of the full $C^{*}$-algebra associated to the free group $\mathbb{F}_{\infty}$. Proc. Edinburgh Math. Soc. 41 (1998), 93-116.

10. M. Junge, Factorization Theory for Spaces of Operators. Habilitation Thesis. Kiel, 1996.

11. M. Junge, Doob's inequality for non-commutative martingales. J. reine angew. Math. 549 (2002), 149-190.

12. M. Junge, Embedding of the operator space $O H$ and the logarithmic 'little Grothendieck inequality'. Invent. Math. 161 (2005), 225-286.

13. M. Junge, Vector-valued $L_{p}$ spaces over QWEP von Neumann algebras. Preprint.

14. M. Junge and H.H. Lee, A Maurey type result for operator spaces. J. Funct. Anal. 254 (2008), 1373-1409.

15. M. Junge and J. Parcet, The norm of sums of independent noncommutative random variables in $L_{p}\left(\ell_{1}\right)$. J. Funct. Anal. 221 (2005), 366-406.

16. M. Junge and J. Parcet, Rosenthal's theorem for subspaces of noncommutatuve $L_{p}$. Duke Math. J. 141 (2008), 75-122.

17. M. Junge and J. Parcet, Operator space embedding of Schatten p-classes into von Neumann algebra preduals. Geom. Funct. Anal. 18 (2008), 522-551.

18. M. Junge and J. Parcet, Mixed-norm inequalities and operator space $L_{p}$ embedding theory. To appear in Mem. Amer. Math. Soc.

19. M. Junge and J. Parcet, A transference method in quantum probability. Preprint.

20. M. Junge and D. Sherman, Noncommutative $L_{p}$ modules. J. Operator Theory 53 (2005), 3-34.

21. M. Junge and Q. Xu, Noncommutative Burkholder/Rosenthal inequalities II: Applications. Israel J. Math. 167 (2008), 227-282.

22. M. Junge and Q. Xu, Representation of certain homogeneuous Hilbertian operator spaces and applications. Preprint.

23. M. Junge and $\mathrm{Q}$. Xu, Interpolation for noncommutative $L_{p}\left(L_{q}\right)$ spaces and applications. In progress. 
24. E. Kirchberg, On nonsemisplit extensions, tensor products and exactness of group $C^{*}-$ algebras. Invent. Math. 112 (1993), 449-489.

25. H. Kosaki, Applications of the complex interpolation method to a von Neumann algebra. J. Funct. Anal. 56 (1984), 29-78.

26. J.L. Krivine, Sous-espaces de dimension finie des espaces de Banach réticulé. Ann. of Math. 104 (1976), 1-29.

27. H.H. Lee, Type and cotype of operator spaces. Studia Math. 185 (2008), 219-247.

28. J. Lindenstrauss and A. Pelczyński, Absolutely summing operators in $L_{p}$-spaces and their applications. Studia Math. 29 (1968), 275-326.

29. B. Maurey, Théorèmes de factorisation pour les opérateurs linéaires à valeurs dans les espaces $L^{p}$. Asterisque 11, 1974.

30. B. Maurey and G. Pisier, Séries de variables aléatoires vectorielles indépendantes et propriétés géométriques des espaces de Banach. Studia Math. 58 (1976), 45-90.

31. J. Parcet, Análisis armónico no conmutativo y geometría de espacios de operadores. Ph. D. Thesis, 2003.

32. J. Parcet, B-convex operator spaces. Proc. Edinburgh Math. Soc. 46 (2003), 649-668

33. J. Parcet and G. Pisier, Non-commutative Khintchine type inequalities associated with free groups. Indiana Univ. Math. J. 54 (2005), 531-556.

34. A. Pietsch, Eigenvalues and s-numbers. Cambridge Studies in Advanced Mathematics, 13. Cambridge University Press, Cambridge, 1987.

35. G. Pisier, Factorization of operators through $L_{p \infty}$ or $L_{p 1}$ and noncommutative generalizations. Math. Ann. 276 (1986), 105-136.

36. G. Pisier, Projections from a von Neumann algebra onto a subalgebra. Bull. Soc. Math. France 123 (1995), 139-153.

37. G. Pisier, Dvoretzky's theorem for operator spaces. Houston J. Math. 22 (1996), 399-416.

38. G. Pisier, The Operator Hilbert Space OH, Complex Interpolation and Tensor Norms. Mem. Amer. Math. Soc. 122 (1996).

39. G. Pisier, Non-Commutative Vector Valued $L_{p}$-Spaces and Completely $p$-Summing Maps. Astérisque 247, 1998.

40. G. Pisier, Introduction to Operator Space Theory. Cambridge University Press, 2003.

41. G. Pisier and D. Shlyakhtenko, Grothendieck's theorem for operator spaces. Invent. Math. 150 (2002), 185-217.

42. Y. Raynaud, On ultrapowers of non-commutative $L_{p}$ spaces. J. Operator Theory 48 (2002), 41-68.

43. H.P. Rosenthal, On subspaces of $L_{p}$. Ann. of Math. 97 (1973), 344-373.

44. Q. Xu, A description of $\left(C_{p}\left[L_{p}(M)\right], R_{p}\left[L_{p}(M)\right]\right)_{\theta}$. Proc. Roy. Soc. Edinburgh Sect. A 135 (2005), 1073-1083.

45. Q. Xu, Operator-space Grothendieck inequalities for noncommutative $L_{p}$-spaces. Duke Math. J. 131 (2006), 525-574.

46. Q. Xu, Embedding of $C_{q}$ and $R_{q}$ into noncommutative $L_{p}$-spaces, $1 \leq p<q \leq 2$. Math. Ann. 335 (2006), 109-131.

47. Q. Xu, Real interpolation approach to Junge's works on embedding of OH and the little Grothendieck inequality. Preprint.

Marius Junge

Department of Mathematics University of Illinois at Urbana-Champaign 1409 W. Green St. Urbana, IL 61891. USA junge@math.uiuc.edu

Javier Parcet Instituto de Ciencias Matemáticas CSIC-UAM-UC3M-UCM

Consejo Superior de Investigaciones Científicas Serrano 121. 28006, Madrid. Spain javier.parcet@uam.es 